\title{
Expressiveness and Nash Equilibrium in Iterated Boolean Games
}

\author{
JULIAN GUTIERREZ, Department of Computer Science, Monash University, Australia \\ PAUL HARRENSTEIN, Department of Computer Science, University of Oxford, UK \\ GIUSEPPE PERELLI, Department of Computer, Automation, and Management Engineering, Sapienza University \\ of Rome, Italy \\ MICHAEL WOOLDRIDGE, Department of Computer Science, University of Oxford, UK
}

\begin{abstract}
We define and investigate a novel notion of expressiveness for temporal logics that is based on game theoretic equilibria of multi-agent systems. We use iterated Boolean games as our abstract model of multi-agent systems [Gutierrez et al. 2013; Gutierrez et al. 2015b]. In such a game, each agent $i$ has a goal $\gamma_{i}$, represented using (a fragment of) Linear Temporal Logic (LTL). The goal $\gamma_{i}$ captures agent $i$ 's preferences, in the sense that the models of $\gamma_{i}$ represent system behaviours that would satisfy $i$. Each player controls a subset of Boolean variables $\Phi_{i}$, and at each round in the game, player $i$ is at liberty to choose values for variables $\Phi_{i}$ in any way that she sees fit. Play continues for an infinite sequence of rounds, and so as players act they collectively trace out a model for LTL, which for every player will either satisfy or fail to satisfy their goal. Players are assumed to act strategically, taking into account the goals of other players, in an attempt to bring about computations satisfying their goal. In this setting, we apply the standard game-theoretic concept of (pure) Nash equilibria. The (possibly empty) set of Nash equilibria of an iterated Boolean game can be understood as inducing a set of computations, each computation representing one way the system could evolve if players chose strategies that together constitute a Nash equilibrium. Such a set of equilibrium computations expresses a temporal property—which may or may not be expressible within a particular LTL fragment. The new notion of expressiveness that we formally define and investigate is then as follows: what temporal properties are characterised by the Nash equilibria of games in which agent goals are expressed in fragments of LTL? We formally define and investigate this notion of expressiveness and related issues, for a range of LTL fragments. For example, a very natural question is the following. Suppose we have an iterated Boolean game in which every goal is represented using a particular fragment $\mathscr{L}$ of LTL: then is it always the case that the equilibria of the game can be characterised within $\mathscr{L}$ ? We show that this is not true in general.
\end{abstract}

CCS Concepts: •Theory of computation $\rightarrow$ Logic; •Computing methodologies $\rightarrow$ Artificial intelligence; Knowledge representation and reasoning; Multi-agent systems;

Additional Key Words and Phrases: logic, game theory, Nash equilibrium, concurrent games, multi-agent systems, expressiveness

ACM Reference Format:

authors, 2019. title. ACM Trans. Comput. Logic V, N, Article 1 (January 2019), 34 pages.

DOI : http://dx.doi.org/10.1145/XXX

\section{INTRODUCTION}

Temporal logics are probably the most successful and widely used class of formalisms for the specification and verification of computer systems [Emerson 1990]. In particular, temporal logics have proven to be enormously valuable in model checking, where a standard question is whether all computations of a given system satisfy a particular temporal logic property $\varphi$ [Clarke et al. 2000]. A natural question relating to temporal logics is that of their expressive power: what system properties is it possible to express within a particular temporal logic or temporal logic fragment? For example, the relative expressiveness of linear versus branching time temporal logics was a major research topic in theoretical computer science for more than a decade, and still generates some debate to the present day [Emerson and Halpern 1986; Vardi 2001].

Author's address: Department of Computer Science, University of Oxford, Oxford OX1 3QD, UK.

Permission to make digital or hard copies of all or part of this work for personal or classroom use is granted without fee provided that copies are not made or distributed for profit or commercial advantage and that copies bear this notice and the full citation on the first page. Copyrights for components of this work owned by others than ACM must be honored. Abstracting with credit is permitted. To copy otherwise, or republish, to post on servers or to redistribute to lists, requires prior specific permission and/or a fee. Request permissions from permissions@acm.org.

(C) 2019 ACM. 1529-3785/2019/01-ART1 $\$ 15.00$

DOI : http://dx.doi.org/10.1145/XXX 
In this article, we are interested in the use of temporal logic for reasoning about multi-agent systems, and in particular, we are interested in questions relating to expressiveness that arise in such settings. We use iterated Boolean games as our abstract model of multi-agent systems [Gutierrez et al. 2013; Gutierrez et al. 2015b]. In this model, each agent exercises exclusive control over a subset of Boolean variables, and the game is played over an infinite number of rounds, where at each round each player chooses a valuation for their variables. The result of play is an infinite computation, which can be understood as a model for Linear Temporal Logic (LTL) [Pnueli 1977; Emerson 1990]. To represent agent preferences in iterated Boolean games, each player $i$ is assumed to have a goal $\gamma_{i}$, expressed using (a fragment of) LTL: the models of $\gamma_{i}$ represent computations that would satisfy $i$. Each player is assumed to act strategically, taking into account the goals of other players, in order to try to bring about computations that will satisfy their goal. For this setting we use the standard game-theoretic concept of Nash equilibrium [Osborne and Rubinstein 1994]: the Nash equilibria of an iterated Boolean game can be understood as characterising a (possibly empty) set of computations, with each computation representing one way the system could evolve if players in the game chose strategies in equilibrium.

Our main interest in the present paper is as follows. Suppose we have a game $G$ in which each player $i$ has a goal $\gamma_{i}$ expressed in a fragment $\mathscr{L}$ of LTL. Then, what temporal property is expressed by the equilibria of $G$ ? In particular, it is very natural to ask whether the equilibria of a game with the players' goals given by formulas in $\mathscr{L}$ can be characterised within $\mathscr{L}$ itself. We formally define and investigate this novel notion of expressiveness, which we refer to as expressiveness in equilibrium. We do this for a range of known fragments of LTL, in particular, the maximal stutter-invariant fragment without a next-operator.

The problem of reasoning about Nash equilibria of concurrent games has, of course, been considered elsewhere. For instance, a popular approach is to develop new formalisms for representing temporal properties of Nash equilibria, and similar game-theoretic solution concepts, in the object language for example by adding new operators to existing temporal logics [Bulling et al. 2008; Gutierrez et al. 2014; Gutierrez et al. 2017a]. Alternatively, one might use a very general formalism such as Strategy Logic to reason about equilibrium properties [Chatterjee et al. 2010]. Our approach-focussing on the temporal properties that Nash equilibrium can distinguish in logicbased (Boolean) games - is fundamentally different. In the present work, we are not concerned with questions such as whether an LTL formula holds on some or all Nash equilibrium computations. Rather, we consider the extent to which Nash equilibria of a game can be characterised using (fragments of) LTL.

Notice that the question of which properties can be expressed by a particular LTL fragment in equilibrium should be distinguished from the question whether there is a formula of the fragment that characterises Nash equilibrium runs. The latter question asks whether there is a formula $\varphi$ (or formula scheme) that holds exactly on the computations sustained by a Nash equilibrium in every Boolean game $G$. The former is the question whether for every formula $\varphi$ we can find a game $G$ such that $\varphi$ is satisfied on exactly those computations of $G$ that are sustained by an equilibrium.

As a motivating example, consider the following temporal variation of the well-known Battle of the Sexes game [Luce and Raiffa 1957], which we will refer to as Boolean Ballet.

Example 1.1 (Boolean Ballet). Suppose that two friends, whom we denote by $i$ and $j$, go out every weekend. Both are dance aficionados and each weekend, either of them has to decide individually whether to go to the ballet or to the discotheque, leading to a sequence of evenings going out. We say they go out together, if they decide to go to the same venue. Assume furthermore that $i$ wishes always to go out together, be it to the ballet or to the disco, whereas $j$ wants to go out to the ballet with $i$ sometimes, but also wants to go to the disco alone at some other occasions.

In this game, the sequence in which $i$ and $j$ go to the ballet the first weekend and to the disco ever after is sustained by a Nash equilibria, but the sequence where they go to the ballet the first two weekends and to the disco ever after is not. As a matter of fact, the Nash equilibria of this game give 


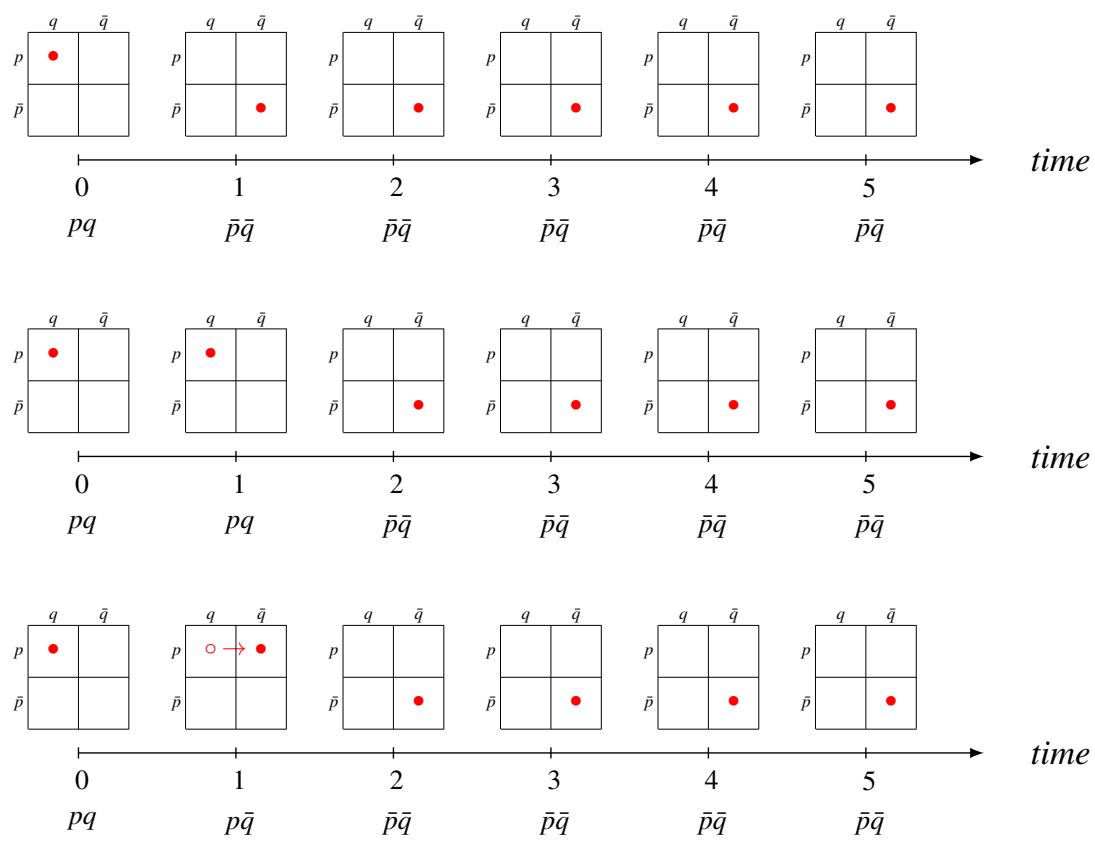

Fig. 1. Three plays of Boolean Ballet, where, at each time (weekend), the red dot indicates how the friends have decided which venue ( $p$ and $q$ for ballet, and $\bar{p}$, respectively, $\bar{q}$ for discotheque) to go to. The first run is sustained by a Nash equilibrium where $i$ 's goal is satisfied and $j$ cannot profitably deviate. The second run is not sustained by a Nash equilibrium. To see this, observe that player $j$ would like to deviate from any strategy profile underlying this run by playing along at time 0 , only to sneak off to the discotheque in the second, leaving $i$ alone stranded at the ballet. This is indicated by the third play.

rise to precisely those sequences of evenings out where $i$ and $j$ always go out together, but at most once to the ballet.

We find that this situation can be conveniently modelled as an iterated Boolean game, for instance, by giving $i$ and $j$ each control over a propositional variable, $p$ and $q$, respectively and assuming that by setting their variable to true at a particular time, the respective friend goes to the ballet and to the disco otherwise. The two players' (binary) preferences can then be expressed using the temporal operators $\mathrm{F}$ ("eventually") and G ("always") only: Player $i$ 's goal could for instance be represented by the LTL-formula $\mathrm{G}(p \leftrightarrow q)$ and player $j$ 's by $\mathrm{F}(p \wedge q) \wedge \mathrm{F}(p \wedge \neg q)$. Also see Figure 1 .

The remarkable feature of Boolean Ballet is that the players goals are invariant under repetition of evenings going out, whereas the set of sequences the Nash equilibria give rise to is not. This means, for instance, that $i$ is just as satisfied when the friends go out together to the ballet and the disco on alternate weekends, as $i$ would be if they were to go out, alternately, to the ballet two weekends in a row and then to the disco two weekends in a row. By contrast, the two friends going out together to the ballet the first weekend and to the disco ever after is sustained by a Nash equilibrium, but going out to the ballet the first two weekends and to the disco every after is not! This phenomenon indicates that, for a given fragment of LTL, the traditional concept of expressiveness for temporal logics can be quite different from our notion of expressiveness in equilibrium. We will therefore explore in detail the issue which temporal properties are characterised by the Nash equilibria of iterated Boolean games with the players' preferences formulated in various fragments of linear temporal logic. 
Our work also has a natural bearing on settings where a designer uses temporal logic to specify the desired behaviour of a system. Consider, for instance, a situation in which a designer is to design a multi-agent system that is to behave accordingly to a given complex specification, and she has to distribute over several agents the complex task represented by the specification. Moreover, these agents may possess only limited computational capabilities, and the designer may want to allocate them tasks that are as "simple" as possible. In our setting, this translates to the designer's specification possibly being formulated in an expressive but possibly computationally expensive fragment of LTL, and the agents having to be assigned objectives phrased in weaker fragments with better computational properties. The designer's task is then to find such "weak" goals for the agents so that the specification is satisfied in those Nash equilibrium computations of the multi-agent system that satisfy the specification. The following simple example illustrates this idea.

Example 1.2 (The Rabbit Hunt). Consider the following coordination task for two agents, $i$ and $j$ who have to catch a rabbit in the dark. One agent holds a torch and the other one has a gun. At each time, the first agent, $i$, can light the torch $(p)$ or not $(\neg p)$, and the second agent, $j$, can fire the gun $q$ or refrain from doing so $(\neg q)$. If $i$ lights the torch without $j$ firing the gun, the rabbit will be alarmed and dash off. Similarly, if $j$ fires the gun without $i$ lighting the torch, agent $j$ will miss. The only way the two of them can catch the rabbit if they light the torch and fire the gun at the same time. This situation can be modelled as one where a system designer aims to fulfil the overall specification that the torch and the gun should not be put to use until a certain time when the torch should be lighted and the gun be fired simultaneously. This specification can be formulated by the temporal logic formula $\gamma_{0}=(\neg p \wedge \neg q) \mathrm{U}(p \wedge q)$, where $\mathrm{U}$ is the until-operator. Assuming that the computational powers of the two agents are limited and that they can only perform tasks specified either as safety goals or as reachability goals, which only contains the always-operator G and the eventuality-operator $\mathrm{F}$ as temporal connectives.

Now, the system designer can find an implementation of the specification $\gamma_{0}$ by assigning to player $i$ objective $\gamma_{i}=\mathrm{F} p$ and to player $j$ objective $\gamma_{j}=\mathrm{G}(p \leftrightarrow q)$. Then, as $\gamma_{0}, \gamma_{i}$, and $\gamma_{j}$ are consistent and neither $i$ nor $j$ would like to deviate from any run satisfying their goals, there clearly is a Nash equilibrium run in which $\gamma_{0}$ is satisfied. Moreover, there are no Nash equilibria in which $\gamma_{0}$ does not hold. Observe that player $i$ can achieve his goal on his own by simply lighting the torch (that is, setting $p$ to true) at some point. Accordingly, this will also happen in all equilibria at some time. Moreover, due to presuppositions inherent in the definition of Nash equilibrium, we may assume that player $j$ knows player $i$ 's strategy and can at each time predict whether $i$ is going to light the torch or not, that is, which truth-value of $p$ assumes, and choose the value for $q$ accordingly. Thus, in every equilibrium run also player $j$ 's goal $\gamma_{j}$ will be satisfied. Now, let $\rho$ be an equilibrium run and $t$ the earliest time that $i$ lights the torch, that is, the first time that $p$ is set to true. Then, simultaneously, $j$ fires the gun, that is, $q$ is set to true at $t$ as well. Moreover, at all previous times, $i$ does not light the torch and neither is the gun fired as we know that player $j$ will have her goal achieved. It follows that the specification $\gamma_{0}$ holds in all equilibria.

Clearly, there are also runs satisfying $\gamma_{0}$ that are not sustained by an equilibrium, for instance, every run in which both $p$ and $q$ hold at time $t=0$ and $p \wedge \neg q$ at some later time. In that case, player $j$ would like to deviate, so as to match the truth values of $p$ and $q$ at each time. This simply means that the specification $\gamma_{0}$ is weaker than its implementation in Nash equilibria.

It may furthermore be worthwhile to observe that the conjunction of the players' goals $\gamma_{i} \wedge \gamma_{j}=$ $\mathrm{F} p \wedge \mathrm{G}(p \leftrightarrow q)$ implies the specification $\gamma_{0}$, but that nevertheless by allocating this formula to both players, there are equilibrium runs that do not satisfy $\gamma_{0}$. For instance, if the players were always to (myopically) set $p$ and $q$ to false, respectively.

Our paper is organised as follows. Following this introduction, we present the background technical concepts used throughout the paper. In Section 3, we present the model of iterated Boolean games and provide a useful characterisation of the computations that are induced by their Nash equilibria. Our main contributions are to be found in Section 4, where we introduce the central concept of expressiveness in equilibrium, explore its ramifications for the full fragment LTL, propositional 
calculus, and the important maximal stutter-invariant fragment without the next-operator. On basis of the game of Boolean Ballet (Example 1.1), we formally demonstrate that the temporal property expressed by a Boolean game with players' goals in a fragment of LTL need not necessarily be expressible in that fragment itself. This is also the case for the maximal stutter-invariant fragment, even though we also show that there are still LTL-properties that this fragment cannot express in equilibrium. This is in contrast to the full LTL-fragment, for which we can show that every nonempty temporal property expressible in full LTL is also expressed by an iterated Boolean game with LTL goals, and vice versa. In Section 5, we study the contrasts between the weaker concepts of projective expressiveness and projective expressiveness in equilibrium. We prove that both full LTL and the maximal stutter-invariant fragment can projectively express in equilibrium every $\omega$-regular temporal property. In Section 6, we argue how another weakening of the regular expressiveness notion, which we refer to as weak expressiveness in equilibrium, chimes in well with the incentive engineering perspective illustrated by the Rabbit Hunt (Example 1.2). We show how the very weak fragment $\mathscr{L}_{\mathrm{X} . \mathrm{F}^{+}}$, which only allows reachability goals to be formulated, can already weakly projectively express in equilibrium every $\omega$-regular temporal property. We conclude by reviewing the related literature (Section 7) and suggesting a number of topics for future research (Section 8).

\section{PRELIMINARIES}

In this section, we provide the necessary preliminaries, introducing the central formal concepts, notions, and definitions we will employ throughout the paper.

\subsection{Words and Languages}

Let $\Sigma$ be an alphabet set of symbols or letters $a, b, c, \ldots$ A word $w$ is finite sequence $w=a_{0} \ldots a_{n}$ of letters and an $\omega$-word is an infinite sequence $w=a_{0} a_{1} a_{2}, \ldots$ of letters. By $\varepsilon$ we denote the empty word. A language is subset $L$ of words over $\Sigma$ and an $\omega$-language is a subset of $\omega$-words over $\Sigma$. For words $w$ and $w^{\prime}$ over an alphabet $\Sigma$ we use the operations of concatenation $\left(w w^{\prime}\right)$, finite iteration $\left(w^{*}\right)$, and infinite iteration $\left(w^{\omega}\right)$. For integers $k \geq 0$, we have $a^{k}$ denote the $k$-fold iteration of symbol $a$. The $\omega$-regular expressions over the alphabet $\Sigma$ are given by the $\omega$-regular expressions constructed by concatenation $(X ; Y)$, union $(X+Y)$, finite iteration $\left(X^{*}\right)$, and infinite iteration $\left(X^{\omega}\right)$, where $X$ and $Y$ are sets of words or $\omega$-words.

An $\omega$-language $L$ over alphabet $\Sigma$ is stutter-invariant if for all $\omega$-words $w=a_{0} a_{1} a_{2} a_{3} \ldots$ and every sequence $k_{0}, k_{1}, k_{2}, \ldots$ of positive integers, $a_{0} a_{1} a_{2} a_{3} \ldots \in L$ if and only if $a_{0}^{k_{0}} a_{1}^{k_{1}} a_{2}^{k_{2}} a_{3}^{k_{3}} \ldots \in$ $L$, where $v^{k}$ denotes the $k$-fold iteration of $v$.

A finite word $w=a_{0} \ldots a_{k}$ is a prefix of a finite word $w=b_{0} \ldots b_{m}$ with $k \leq m$ or an $\omega$-word $w^{\prime}=b_{0} b_{1} b_{2} b_{3} \ldots$ if $a_{t}=b_{t}$ for all $t \leq k$. The set of prefixes of an $\omega$-word $w$ we denote by $\operatorname{prefix}(w)$. We say that a finite word $w$ is the (unique) maximal common prefix of $\omega$-words $w^{\prime}$ and $w^{\prime \prime}$ if $w$ is a prefix of both $w^{\prime}$ and $w^{\prime \prime}$ and no prefix of greater length has this property. For instance, $a b$ and $a b c$ are both common prefixes of the $\omega$-words $a b c c c c .$. and $a b c d d d \ldots$, but only $a b c$ is maximal.

For an excellent survey of infinite words, see [Perrin and Pin 2004].

\subsection{Propositional Temporal Logic}

We make extensive use of Linear Temporal Logic and the iterated Boolean games based on it. In this section, we present the core concepts of these frameworks and a number of auxiliary notions.

Linear Temporal Logic (LTL). We use the well-known framework of Linear Temporal Logic (LTL) [Pnueli 1977; Emerson 1990; Thomas 1990; Baier and Katoen 2008; Demri et al. 2016]. The formulas of LTL are constructed in the usual fashion from a non-empty and finite set $\Phi$ of propositional variables $p, q, r, \ldots$ using the Boolean connectives negation $(\neg \varphi)$ and disjunction $(\varphi \vee \psi)$, as well as the temporal operators next $(\mathrm{X} \varphi)$, eventually $(\mathrm{F} \varphi)$, always $(\mathrm{G} \varphi)$, and until $(\varphi \cup \psi)$. The connectives Truth $(\top)$, falsity $(\perp)$, conjunction $(\varphi \wedge \psi)$, implication $(\varphi \rightarrow \psi)$, and bi-implication $(\varphi \leftrightarrow \psi)$, are introduced as the usual abbreviations of $p \vee \neg p, \neg \top, \neg(\neg \varphi \vee \neg \psi)$, $\neg \varphi \vee \psi$, and $(\varphi \rightarrow \psi) \wedge(\psi \rightarrow \varphi)$, respectively. Where $p$ is a propositional variable we sometimes 
write $\bar{p}$ for $\neg p$. We also sometimes omit conjunctions in conjunctive clauses and, for instance, denote $p \wedge \neg q \wedge r$ by $p \bar{q} r$.

By a valuation $v$ we understand a subset of propositional variables, that is, $v \subseteq \Phi$. Thus the set of valuations over $\Phi$ is given by $2^{\Phi}$. Intuitively, a propositional variable $p$ is set to true at valuation $v$ if $p \in v$, and false otherwise. For a valuation $v \subseteq \Phi$, we have $\chi_{v}^{\Phi}$ denote the characteristic clause for $v$ given by $\chi_{v}^{\Phi}=\bigwedge_{p \in v} p \wedge \bigwedge_{q \in \Phi \backslash v} \bar{q}$. Thus, for $w, v \subseteq \Phi$, we have $w \models \chi_{v}^{\Phi}$ if and only if $v=w$. We will also identify valuations and their characterising clauses. Accordingly, we, for instance, write $p \bar{q} r$ for valuation $\{p, r\}$ if $\Phi=\{p, q, r\}$.

The formulas of LTL are interpreted with respect to runs $\rho=v_{0} v_{1} v_{2} v_{3} \ldots$, which we define as infinite sequences (or $\omega$-words) over valuations in $\Phi$, that is, $\rho \in\left(2^{\Phi}\right)^{\omega}$. We denote the set of runs over valuations in $2^{\Phi}$ by runs , again omitting the reference to $\Phi$ when clear from the context. Thus every LTL formula defines an $\omega$-language over $2^{\Phi}$, which could also be characterised by other means, for instance, by automata or grammars.

The semantics of LTL then interprets LTL-formulas with respect to a run $\rho=v_{0} v_{1} v_{2} v_{3} \ldots$ and time index $t \in \mathbb{N}$ as follows.

$$
\begin{aligned}
& \rho, t \models p \quad \text { iff } \quad p \in v_{t} \quad(\text { for } p \in \Phi) \\
& \rho, t \models \neg \varphi \quad \text { iff } \quad \rho, t \mid \neq \varphi \\
& \rho, t=\varphi \vee \psi \quad \text { iff } \quad \rho, t=\varphi \text { or } \rho, t=\psi \\
& \rho, t \models \mathrm{X} \varphi \quad \text { iff } \quad \rho, t+1 \models \varphi \\
& \rho, t=\mathrm{F} \varphi \quad \text { iff } \quad \rho, t^{\prime}=\varphi \text { for some } t^{\prime} \geq t \\
& \rho, t=\mathrm{G} \varphi \quad \text { iff } \quad \rho, t^{\prime}=\varphi \text { for all } t^{\prime} \geq t \\
& \rho, t=\varphi \cup \psi \quad \text { iff } \quad \text { for some } t^{\prime} \geq t \text { both } \rho, t^{\prime} \mid=\psi \text {, and } \rho, t^{\prime \prime} \models \varphi \text { for all } t \leq t^{\prime \prime}<t^{\prime}
\end{aligned}
$$

We say that a run $\rho$ satisfies a formula $\varphi$ if $\rho, 0=\varphi$. The set of runs in $r u n s_{\Phi}$ that satisfy formula $\varphi$ we denote by $\operatorname{runs}_{\Phi}(\varphi)$. A formula $\varphi$ is satisfiable if some run satisfies $\varphi$. Observe that $\neg \mathrm{X} \varphi$ is equivalent to $\mathrm{X} \neg \varphi$, and $\mathrm{F} \varphi$ to $\neg \mathrm{G} \neg \varphi$.

We also employ a number of auxiliary concepts. For $\Psi \subseteq \Phi$ and $\rho \in \operatorname{runs}_{\Phi}$, we write $\left.\rho\right|_{\Psi}$ for the restriction (or projection) of $\rho$ to $\Psi$, that is, if $\rho=v_{1}, v_{2}, \ldots$ then $\left.\rho\right|_{\Psi}=w_{1}, w_{2}, \ldots$ where $w_{t}=$ $v_{t} \cap \Psi$ for each $t \geq 1$. For $X \subseteq$ runs $_{\Phi}$ and $\Psi \subseteq \Phi$, we denote by $\left.X\right|_{\Psi}$ the set $\left\{\left.\rho\right|_{\Psi} \in\right.$ runs $\left.\Psi: \rho \in X\right\}$.

By a history we understand a finite and possibly empty sequence $\pi=v_{0}, \ldots, v_{k}$ in $\left(2^{\Phi}\right)^{*}$. We let length $(\pi)$ denote the length of $\pi$.

Fragments of Linear Temporal Logic. We study the expressive power of the most natural, and therefore most widely known, fragments of LTL. Such fragments are the "stutter-invariant" fragment (technically, the $\mathrm{X}$-free fragment), denoted by $\mathscr{L}_{\mathrm{U}}$, as well as other fragments where the use of the "until" operator is restricted to simply being G or F, leading to the following sublogics: $\mathscr{L}_{\mathrm{G}, \mathrm{F}, \mathrm{X}}$ (sometimes also referred to as "restricted LTL", for instance, by [Perrin and Pin 2004]), where only $\mathrm{G}$ and $\mathrm{F}$ and $\mathrm{X}$ are allowed, and with similar interpretations, the sublogics $\mathscr{L}_{\mathrm{G}, \mathrm{F}}, \mathscr{L}_{\mathrm{X}, \mathrm{G}^{+}}$, and $\mathscr{L}_{\mathrm{X}, \mathrm{F}^{+}}$, where the "+" notation indicates that negations are allowed only in front of propositional variables (otherwise, for instance, the $\mathscr{L}_{\mathrm{G}}$ fragment would be the same as the $\mathscr{L}_{\mathrm{F}}$ fragment). The fragment $\mathscr{L}_{\mathrm{G}, \mathrm{F}}$ was briefly discussed in Example 1.1. We will not study the extremely weak sublogics $\mathscr{L}_{\mathrm{G}^{+}}, \mathscr{L}_{\mathrm{F}^{+}}, \mathscr{L}_{\mathrm{X}}$, and $\mathscr{L}_{\varnothing}$ (the latter referring to propositional logic). On the one hand, the two latter sublogics cannot express interesting properties of infinite runs-all their models are finite words; on the other hand, the first two sublogics can express only very limited classes of temporal properties: only "safety" properties in the case of $\mathscr{L}_{\mathrm{G}^{+}}$and only "reachability" properties in the case of $\mathscr{L}_{\mathrm{F}^{+}}$. Indeed, our study covers the most relevant LTL fragments in the literature. Finally, by $\mathscr{L}_{\omega \text {-reg }}$ we refer to the set of $\omega$-regular expressions, which are seen as an extension of LTL, rather than a fragment [Wolper 1983]. Sometimes we refer explicitly to the set $\Phi$ of variables over which $\mathscr{L}$ is defined, and write $\mathscr{L}(\Phi)$ for $\mathscr{L}$.

For a detailed discussion and comparison of expressiveness of the various fragments of LTL, also see [Strejček 2004]. 


\section{ITERATED BOOLEAN GAMES AND NASH EQUILIBRIUM}

Boolean games were introduced by [Harrenstein et al. 2001] and further developed by, among others, Bonzon, Lang, and Wooldridge [Bonzon et al. 2006; Endriss et al. 2011]. In this paper, we adopt the framework of iterated Boolean games as proposed by [Gutierrez et al. 2015b], where players play a Boolean game over an infinite number of rounds and where each players's goal is given by an LTL-formula.

\subsection{Iterated Boolean Games}

For $\mathscr{L}$ a fragment of LTL over $\Phi$, an $\mathscr{L}$-iterated Boolean game (over $\Phi$ ) is a tuple

$$
G=\left(N, \Phi, \Phi_{1}, \ldots, \Phi_{n}, \gamma_{1}, \ldots, \gamma_{n}\right),
$$

where $N$ is a set of players, each $\Phi_{i} \subseteq \Phi$ is a subset of propositional variables under control of player $i$, and $\gamma_{i}$ is a formula in $\mathscr{L}$ representing player $i$ 's preferences over runs $s_{\Phi}$. We assume $\Phi_{1}, \ldots, \Phi_{n}$ to partition $\Phi$, that is, $\Phi_{1} \cup \cdots \cup \Phi_{n}=\Phi$ and $i \neq j$ implies $\Phi_{i} \cap \Phi_{j}=\emptyset$. Henceforth, we also refer to an $\mathscr{L}$-iterated Boolean game as an $\mathscr{L}$-game.

Example 3.1 (Boolean Ballet, cont'd). The game Boolean Ballet game in the introduction can thus be formalised as the tuple $G_{b b}=\left(\{i, j\},\{p, q\}, \Phi_{i}, \Phi_{j}, \gamma_{i}, \gamma_{j}\right)$, where $\Phi_{i}=\{p\}$ and $\Phi_{j}=\{p\}$, as well as $\gamma_{i}=\mathrm{G}(p \leftrightarrow q)$ and $\gamma_{j}=\mathrm{F} p q \wedge \mathrm{F} p \bar{q}$.

An iterated Boolean game takes place in an infinite number of rounds and in every round each player $i$ simultaneously makes a choice $v_{i} \subseteq \Phi_{i}$ of values for the propositional variables under its control based on the values chosen by all players in previous rounds. Formally, a strategy for a player $i$ is a function $f_{i}:\left(2^{\Phi}\right)^{*} \rightarrow 2^{\Phi_{i}}$ which associates with every history $\pi \in\left(2^{\Phi}\right)^{*}$ a choice $f_{i}(\pi) \in$ $2^{\Phi_{i}}$. A strategy profile is a tuple $f=\left(f_{1}, \ldots, f_{n}\right)$ that associates with each player $i$ a strategy $f_{i}$ and induces an infinite run $\rho(f)=v_{0} v_{1} v_{2} v_{3} \ldots$ defined as follows:

$$
\begin{aligned}
v_{0} & =f_{1}(\varepsilon) \cup \cdots \cup f_{n}(\varepsilon) \\
v_{t+1} & =f_{1}\left(v_{0}, \ldots, v_{t}\right) \cup \cdots \cup f_{n}\left(v_{0}, \ldots, v_{t}\right)
\end{aligned}
$$

With a slight abuse of notation, we also write $f(\varepsilon)=v_{0}$ and $f\left(v_{0} \ldots v_{t}\right)=v_{t+1}$.

A strategy $f_{i}$ as defined above can make player $i$ 's choice at time $t$ dependent on the preceding path $v_{0} \ldots v_{t-1}$. It seems, however, natural to assume a player can also simply choose to play a sequence of valuations in $\Phi_{i}$ no matter what choices the other players make. Such strategies have much less structure. Formally, we say that a player's strategy $f_{i}$ is naive if $f_{i}\left(v_{0} \ldots v_{t}\right)=f_{i}\left(v_{0}^{\prime} \ldots v_{t}^{\prime}\right)$ for all $t \geq 0$, all paths $v_{0} \ldots v_{t}$ and $v_{0}^{\prime} \ldots v_{t}^{\prime}$ of equal length.

A player $i$ strictly prefers runs that satisfy $\gamma_{i}$ to runs that do not and is indifferent otherwise, that is, $i$ strictly prefers run $\rho$ to run $\rho^{\prime}$ if and only if $\rho=\gamma_{i}$ and $\rho^{\prime} \not=\gamma_{i}$. A player $i$ (weakly) prefers run $\rho$ to run $\rho^{\prime}$ if it is not the case that $i$ strictly prefers run $\rho^{\prime}$ to $\rho$. Thus, each player's preferences in iterated Boolean games are dichotomous, dividing the set of runs into those that are preferred and those that are not preferred.

\subsection{Nash Equilibrium and Equilibrium Runs}

It can easily be seen that with the players, strategies and preferences defined in this way, each iterated Boolean game defines a strategic game in the game-theoretic sense of the word [Osborne and Rubinstein 1994; Maschler et al. 2013; Shoham and Leyton-Brown 2008]. Accordingly, the usual game theoretic solution concepts are available for the analysis of iterated Boolean games. This in particular holds for (pure) Nash equilibrium, which in our present setting is a strategy profile $f^{*}=\left(f_{1}^{*}, \ldots, f_{n}^{*}\right)$ such that for all players $i$ and all of $i$ 's strategies $g_{i}$, we have that

$$
\rho\left(f_{-i}^{*}, g_{i}\right) \models \gamma_{i} \text { implies } \rho\left(f^{*}\right) \models \gamma_{i},
$$

where $\left(f_{-i}^{*}, g_{i}\right)$ denotes the profile $\left(f_{1}^{*}, \ldots, f_{i-1}^{*}, g_{i}, f_{i+1}^{*}, \ldots, f_{n}^{*}\right)$. 
We say a Nash equilibrium $f^{*}=\left(f_{1}^{*}, \ldots, f_{n}^{*}\right)$ is naive if every strategy $f_{i}^{*}$ is naive. We also have the following useful fact, which states that to establish whether a strategy profile is a Nash equilibrium, it suffices to investigate if it does not allow profitable deviations to naive strategies.

LEMMA 3.2. Let $G=\left(N, \Phi_{1}, \ldots, \Phi_{n}, \gamma_{1}, \ldots, \gamma_{n}\right)$ be an iterated Boolean game. Then a strategy profile $f=\left(f_{1}, \ldots, f_{n}\right)$ is a Nash equilibrium if and only if $\rho\left(f_{-i}^{*}, g_{i}\right) \models \gamma_{i}$ implies $\rho\left(f^{*}\right) \models \gamma_{i}$ for all players $i$ and all naive strategies $g_{i}$.

Proof. The "only if"-direction is trivial. For the "if"-direction, assume that $f=\left(f_{1}, \ldots, f_{n}\right)$ is not a Nash equilibrium. Then, there is some player $i$ and some strategy $g_{i}$ such that $\rho(f) \not \neq \gamma_{i}$ and $\rho\left(f_{-i}, g_{i}\right) \models \gamma_{i}$. Let $\rho\left(f_{-i}, g_{i}\right)=w_{0} w_{1} w_{2}, \ldots$. Now define the naive strategy $g_{i}^{\prime}$ for player $i$ such that $g_{i}^{\prime}(\varepsilon)=g_{i}(\varepsilon)$ and $g_{i}^{\prime}\left(v_{0} \ldots v_{t}\right)=g_{i}\left(w_{0}, \ldots, w_{t}\right)$ for every history $v_{0} \ldots v_{t}$. By a straightforward inductive argument it can then easily be established that $\rho\left(f_{-i}, g_{i}\right)=\rho\left(f_{-i}, g_{i}^{\prime}\right)$. It thus follows that $\rho\left(f_{-i}, g_{i}^{\prime}\right) \models \gamma_{i}$ and $\rho(f) \not \models \gamma_{i}$ for some player $i$ and some naive strategy $g_{i}^{\prime}$, as desired.

We say that a run $\rho \in \operatorname{runs}_{\Phi}$ is sustained by a Nash equilibrium $f^{*}$ in a game $G$ whenever $\rho\left(f^{*}\right)=$ $\rho$. We then refer to $\rho$ as an equilibrium run. The set of equilibrium runs of $G$-rather than the set of equilibria itself-we denote by $N E(G)$.

The relationship between the strategy profiles that are Nash equilibria on the one hand and the equilibrium runs that are sustained by them on the other, is a complex one. Due to the deterministic character of strategies as we defined them, it is obvious that each Nash equilibrium sustains one run only, namely, the run it induces. It is also easy to construct examples showing that iterated Boolean game may have multiple equilibria, which moreover may sustain different runs. Neither should it come as a surprise that one run can be sustained by two different equilibria. However, whether a profile is a Nash equilibrium not only depends on its behaviour on prefixes of the run it induces, but also on its definition on histories that are not a prefix of the equilibrium run. Hence, it is quite possible for two profiles $f=\left(f_{1}, \ldots, f_{n}\right)$ and $g=\left(g_{1}, \ldots, g_{n}\right)$ to induce the same run, that is, $\rho(f)=\rho(g)$, even though $f$ is a Nash equilibrium and $g$ is not. Put slightly differently, the fact that a profile $f=\left(f_{1}, \ldots, f_{n}\right)$ induces an equilibrium run $\rho$, does not imply that $f$ has to be an equilibrium. To see this, consider the following example.

Example 3.3. Consider the following game with two players, $i$ and $j$ controlling variables $p$ and $q$, respectively. Let further the players' goals be given by $\gamma_{i}=\mathrm{G} p q$ and $\gamma_{j}=\mathrm{GF} p \bar{q}$. Now consider the strategy $f_{i}$ for player $i$ defined such that $f_{i}(\varepsilon)=p$ and, for all histories $\pi=w_{0} \ldots w_{k}$ $(k \geq 0)$,

$$
f_{i}\left(w_{0} \ldots w_{k}\right)= \begin{cases}p & \text { if } w_{t}=p q \text { for all } 1 \leq t \leq k \\ \bar{p} & \text { otherwise }\end{cases}
$$

Also consider the naive strategies $g_{i}$ and $g_{j}$, for players $i$ and $j$, respectively, such that $g_{i}(\pi)=p$ and $g_{j}(\pi)=q$ for all histories $\pi$. Then,

$$
\rho\left(f_{i}, g_{j}\right)=\rho\left(g_{i}, g_{j}\right)=v_{0} v_{1} v_{2} v_{3} \ldots,
$$

where $v_{t}=p q$ for all $t \geq 0$. Thus, on this run, player $i$ 's goal is satisfied on $\rho$, but player $j$ 's goal is not. However, $\left(f_{i}, g_{j}\right)$ is a Nash equilibrium, whereas $\left(g_{i}, g_{j}\right)$ is not. To see the latter, observe that player $j$ would like to deviate from $\left(g_{i}, g_{j}\right)$, for instance, by playing the naive strategy $g_{j}^{\prime}$ defined such that $g_{j}^{\prime}(\pi)=\bar{q}$ for all histories $\pi$. Note that $\rho\left(g_{i}, g_{j}^{\prime}\right)=w_{0} w_{1} w_{2} \ldots$ such that $w_{t}=p \bar{q}$ for all $t \geq 0$, and accordingly $\rho\left(g_{i}, g_{j}^{\prime}\right)$ satisfies $j$ 's goal.

To appreciate that $\left(f_{i}, g_{j}\right)$ is a Nash equilibrium, assume for a contradiction that there is some strategy $g_{j}^{\prime \prime}$ for player $j$ such that $\rho\left(f_{i}, g_{j}^{\prime \prime}\right)=u_{0} u_{1} u_{2} \ldots$ satisfies $j$ 's goal $\gamma_{j}$. Then, there is a smallest $t \geq 0$ such that $v_{t} \neq u_{t}$. In particular, $u_{t} \neq p q$. By definition of $f_{i}$ it then follows that $u_{t^{\prime}} \not \models p \bar{q}$ for all $t^{\prime}>t$. Hence, $\rho\left(f_{i}, g_{j}^{\prime \prime}\right) \not \forall \mathrm{GF} p \bar{q}$, that is, $\rho\left(f_{i}, g_{j}^{\prime \prime}\right)$ does not satisfies $j$ 's goal $\gamma_{j}$. Therefore, player $j$ does not want to deviate from $\left(f_{i}, g_{j}\right)$. 
Perhaps more profoundly, we find that that whether a run is sustained by an equilibrium cannot be reduced to this run satisfying particular players' goals and not satisfying those of other players. As the following example demonstrates, it is quite possible for two runs $\rho$ and $\rho^{\prime}$ to satisfy the same players' goals - that is, $\rho \models \gamma_{i}$ if and only if $\rho^{\prime} \models \gamma_{i}$, for all players $i$-even if $\rho$ is sustained by a Nash equilibrium and $\rho^{\prime}$ is not.

Example 3.4. Again consider a two-player game where player $i$ controls variable $p$ and player $j$ variable $q$. Let the players' goals be given by $\gamma_{i}=\mathrm{Fpq}$ and $\gamma_{j}=\top$, respectively. Then, run $\rho=$ $v_{0} v_{1} v_{2} \ldots$ with $v_{t}=\bar{p} \bar{q}$ for all $t \geq 0$ is sustained by the Nash equilibrium where the two players play naive strategies that always set $p$ and $q$ to false. Thus, $\gamma_{2}$ is satisfied, but $\gamma_{1}$ is not. The latter is also true of run $\rho^{\prime}=w_{0} w_{1} w_{2} \ldots$ with $w_{t}=\bar{p} q$ for all $t \geq 0$. This run, however, is not sustained by any Nash equilibrium: for every strategy profile $f=\left(f_{i}, f_{j}\right)$ that induces run $\rho^{\prime}, f_{i}(\pi)=\bar{p}$ and $f_{j}(\pi)=q$ for every prefix $\pi$ of $\rho^{\prime}$. But then, player $i$ would want to deviate to a strategy that sets $p$ to true at some point, that is, to a strategy $g_{i}$ with $g_{i}(\pi)=p$ for some prefix $\pi$ of $\rho^{\prime}$.

We find that, nevertheless, we can obtain a convenient characterisation of the Nash equilibrium runs in an iterated Boolean game. To this end, we first introduce a couple of auxiliary concepts.

In Example 3.3, the crucial difference between player $i$ 's strategies $f_{i}$ and $g_{i}$ is that the former precludes player $j$ achieving his goal no matter which strategy $j$ chooses, whereas the latter does not. In an important sense, by playing $f_{i}$, player $i$ threatens to punish player $j$ if $j$ were to deviate and play a strategy that would result in another run than $\rho\left(f_{i}, g_{j}\right)$. In this way, player $i$ can 'coerce' player $j$ to play $g_{j}$ even if by doing so player $j$ does not achieve his goal: by playing any other strategy player $j$ would not achieve his aim either! Formally, for a given player $j$, we say a run $\rho$ is consistent with a punishment profile against $j$ if there is some strategy profile $f^{j}=\left(f_{1}^{j}, \ldots, f_{n}^{j}\right)$ such that $\rho=\rho\left(f_{1}^{j}, \ldots, f_{n}^{j}\right)$ and $\rho\left(f_{-j}^{j}, g_{j}\right) \not \models \gamma_{j}$ for all strategies $g_{j}$ for player $j$. Observe that for the the punishment of player $j$ to be effective, the other players may have to coordinate their strategies.

Lemma 3.5 below characterises an equilibrium run of an iterated Boolean games as a run $\rho$ that is consistent with a punishment profile against each player that does not achieves his goal $\rho$. Note that this allows for each the punishment profile against each 'losing' player to be different. In the proof we therefore face the challenge to combine a profile $f=\left(f_{1}, \ldots, f_{n}\right)$ that produces a given run $\rho=v_{0} v_{1} v_{2} \ldots$ with the punishment profiles against the losing players, if these are available, into an equilibrium profile $f^{*}=\left(f_{1}^{*}, \ldots, f_{n}^{*}\right)$ that also sustains $\rho$. For every prefix $v_{0} \ldots v_{t}$ of $\rho$, we can define $f_{i}\left(v_{0} \ldots v_{t}\right)=f_{i}^{*}\left(v_{0} \ldots v_{t}\right)$ for each player $i$. If some (losing) player $j$, however, deviates from $f_{j}$, the other players will want to jointly enact a punishment profile $f_{-j}^{j}$ against $j$. Note that, as we are dealing with Nash equilibrium, we are only concerned with individual deviations by a single losing player.

A specific feature of iterated Boolean games is that a player's choice of strategy is immediately reflected in the run that ensues. If $\rho\left(f_{-j}, f_{j}\right)=v_{0} v_{1} v_{2} \ldots$, then we know that $f_{j}(\varepsilon)=v_{0} \cap \Psi_{j}$ and $f_{j}\left(v_{0} \ldots v_{t}\right)=v_{t+1} \cap \Phi_{j}$ for all $t \geq 0$. Accordingly, deviations by a single player can be spotted straightforwardly and immediately: if $\rho(f)=v_{0} v_{1} v_{2} \ldots$ and at some point a history $\pi=v_{0} \ldots v_{t-1} w_{t}$ such that both $w_{t} \cap \Phi_{j} \neq v_{t} \cap \Phi_{j}$ and $w_{i} \cap \Phi_{i}=v_{i} \cap \Phi_{i}$ for all players $i$ distinct from $j$ materialises, it is clear that the deviation from $\rho$ can uniquely be attributed to player $j .{ }^{1}$ Formally, we say that a history $w_{0} \ldots w_{t}$ is a $j$-deviation from $\rho=v_{0} v_{1} v_{2} \ldots$ by player $j$ if there is an $s \leq t$ such that $w_{0} \ldots w_{s-1}=v_{0} \ldots v_{s-1}$ (on the understanding that $w_{0} \ldots w_{s-1}=v_{0} \ldots v_{s-1}=\varepsilon$ if $\left.s=0\right), w_{s} \cap \Phi_{j} \neq$ $v_{s} \cap \Phi_{j}$, and $w_{s} \cap \Phi_{i}=v_{s} \cap \Phi_{i}$ for all players $i$ distinct from $j$, that is, if the first deviation from $\rho$ can uniquely be attributed to $j$.

On this basis, we now obtain the following characterisation of Nash equilibrium runs in iterated Boolean games.

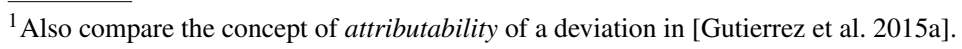


LEMMA 3.5. Let $\rho=v_{0} v_{1} v_{2} \ldots$ be a run in an iterated Boolean game $G$. Then, $\rho \in N E(G)$ if and only if for each player $j$ with $\rho \not \forall \gamma_{j}$, run $\rho$ is consistent with a punishment profile against $j$.

PROOF. For the "only if"-direction assume for contraposition that there is a player $j$ with $\rho \not \models \gamma_{j}$ against whom there is no punishment profile consistent with $\rho$. That is, there is no strategy profile $f^{j}=\left(f_{1}^{j}, \ldots, f_{n}^{j}\right)$ such that $\rho=\rho\left(f_{1}^{j}, \ldots, f_{n}^{j}\right)$ and $\rho\left(f_{-j}^{j}, g_{j}\right) \not \models \gamma_{i}$ for all strategies $g_{j}$. Now consider an arbitrary strategy profile $f=\left(f_{1}, \ldots, f_{n}\right)$ with $\rho=\rho\left(f_{1}, \ldots, f_{n}\right)$. Then, there is some strategy $g_{j}$ for $j$ such that $\left(f_{-j}, g_{j}\right) \models \gamma_{i}$. Accordingly, $f$ is no Nash equilibrium and, with $f$ having been chosen arbitrarily, it follows that $\rho \notin N E(G)$.

For the opposite direction, assume $\rho=v_{0} v_{1} v_{2} \ldots$ to be consistent with a punishment profile $f^{j}=$ $\left(f_{1}^{j}, \ldots, f_{n}^{j}\right)$ against each player $j$ with $\rho \not \models \gamma_{j}$. Let, moreover, $f=\left(f_{1}, \ldots, f_{n}\right)$ be a profile such that $\rho=\rho\left(f_{1}, \ldots, f_{n}\right)$; observe that we may assume that such a profile exists. Now define $f^{*}=$ $\left(f_{1}^{*}, \ldots, f_{n}^{*}\right)$ as the strategy profile such that for every player $i$ and every history $\pi$,

$$
f_{i}^{*}(\pi)= \begin{cases}f_{i}^{j}(\pi) & \text { if } \pi \text { is a } j \text {-deviation from } \rho \text { for some player } j \text { with } \rho \not \models \gamma_{j}, \\ f_{i}(\pi) & \text { otherwise. }\end{cases}
$$

Observe that $f^{*}$ is well defined because by definition each history can be a $j$-deviation from $\rho$ for at most one player $j$ only. As for every player $i$, neither $\varepsilon$ nor any prefix $v_{0} \ldots v_{t}$ of $\rho$ is a $j$-deviation, we have $f_{i}(\varepsilon)=f^{*}(\varepsilon)$ and $f_{i}^{*}\left(v_{0} \ldots v_{t}\right)=f_{i}\left(v_{0} \ldots v_{t}\right)$ for every $t \geq 0$, and, hence, $\rho\left(f^{*}\right)=\rho(f)=$ $\rho$. In a similar vein, observe that consistency with $\rho=v_{0} v_{1} v_{2} \ldots$ implies that $f_{i}^{j}(\varepsilon)=f_{i}(\varepsilon)$ and $f_{i}^{j}\left(v_{0} \ldots v_{t}\right)=f_{i}\left(v_{0} \ldots v_{t}\right)$ for prefix $v_{0} \ldots v_{t}$ of $\rho$ and all players $i$ (including $j$ ).

We conclude the proof by showing that $f^{*}$ is a Nash equilibrium. To this end, consider an arbitrary player $j$ with $\rho\left(f^{*}\right) \not \models \gamma_{j}$. Then, also $\rho(f) \not \neq \gamma_{j}$. Now also consider an arbitrary strategy $g_{j}$ for $j$. We demonstrate that $\rho\left(f_{-j}^{*}, g_{j}\right) \not \forall \gamma_{j}$, which implies that $f^{*}$ is a Nash equilibrium. As $f^{j}=\left(f_{1}^{j}, \ldots, f_{n}^{j}\right)$ is a punishment profile, $\rho\left(f_{-j}^{j}, g_{j}\right) \not \models \gamma_{j}$. Hence, it suffices to show that $\rho\left(f_{-j}^{*}, g_{j}\right)=\rho\left(f_{-j}^{j}, g_{j}\right)$.

To this end, let $\rho\left(f_{-j}^{*}, g_{j}\right)=w_{0} w_{1} w_{2} \ldots$ and $\rho\left(f_{-j}^{j}, g_{j}\right)=u_{0} u_{1} u_{2} \ldots$ We prove by induction that $w_{t}=u_{t}$ for every $t \geq 0$. For the basis $t=0$, observe that $\varepsilon$ is not a $j$-deviation from $\rho$. Hence,

$$
\begin{aligned}
w_{0} & =f_{1}^{*}(\varepsilon) \cup \cdots \cup g_{j}(\varepsilon) \cup \cdots \cup f_{n}^{*}(\varepsilon) \\
& =f_{1}(\varepsilon) \cup \cdots \cup g_{j}(\varepsilon) \cup \cdots \cup f_{n}(\varepsilon) \\
& =f_{1}^{j}(\varepsilon) \cup \cdots \cup g_{j}(\varepsilon) \cup \cdots \cup f_{n}^{j}(\varepsilon) \\
& =u_{0} .
\end{aligned}
$$

For the induction step, we may assume that $w_{0} \ldots w_{t}=u_{0} \ldots u_{t}$ to prove that $w_{t+1}=u_{t+1}$. First assume that $w_{0} \ldots w_{t}=v_{0} \ldots v_{t}$. Then obviously also $u_{0} \ldots u_{t}=v_{0} \ldots v_{t}$. Moreover, $w_{0} \ldots w_{t}$ is a $i$-deviation from $\rho$ for no player $i$. Hence,

$$
\begin{aligned}
w_{t+1} & =f_{1}^{*}\left(w_{0} \ldots w_{t}\right) \cup \cdots \cup g_{j}\left(w_{0} \ldots w_{t}\right) \cup \cdots \cup f_{n}^{*}\left(w_{0} \ldots w_{t}\right) \\
& =f_{1}^{*}\left(v_{0} \ldots v_{t}\right) \cup \cdots \cup g_{j}\left(v_{0} \ldots v_{t}\right) \cup \cdots \cup f_{n}^{*}\left(v_{0} \ldots v_{t}\right) \\
& =f_{1}\left(v_{0} \ldots v_{t}\right) \cup \cdots \cup g_{j}\left(v_{0} \ldots v_{t}\right) \cup \cdots \cup f_{n}\left(v_{0} \ldots v_{t}\right) \\
& =f_{1}^{j}\left(v_{0} \ldots v_{t}\right) \cup \cdots \cup g_{j}\left(v_{0} \ldots v_{t}\right) \cup \cdots \cup f_{n}^{j}\left(v_{0} \ldots v_{t}\right) \\
& =i . h f_{1}^{j}\left(u_{0} \ldots u_{t}\right) \cup \cdots \cup g_{j}\left(u_{0} \ldots u_{t}\right) \cup \cdots \cup f_{n}^{j}\left(u_{0} \ldots u_{t}\right) \\
& =u_{t+1} .
\end{aligned}
$$

Finally, assume that $w_{0} \ldots w_{t} \neq v_{0} \ldots v_{t}$. Consider the smallest $0 \leq s \leq t$ such that $w_{s} \neq u_{s}$. Then, $w_{0} \ldots w_{s-1}=v_{0} \ldots v_{s-1}$, on the understanding that $w_{0} \ldots w_{s-1}=\varepsilon$ if $s=0$. Accordingly, $w_{0} \ldots w_{s-1}$ is a $k$-deviation from $\rho$ for no player $k$. Hence, for all $i$ distinct from $j$,

$$
f_{i}^{*}\left(w_{0} \ldots w_{s-1}\right)=f_{i}\left(w_{0} \ldots w_{s-1}\right)=f_{i}\left(v_{0} \ldots v_{s-1}\right)=v_{s} \cap \Phi_{i}
$$


It follows that $w_{0} \ldots w_{t}$ is a $j$-deviation from $\rho$. Consequently,

$$
\begin{aligned}
w_{t+1} & =f_{1}^{*}\left(w_{0} \ldots w_{t}\right) \cup \cdots \cup g_{j}\left(w_{0} \ldots w_{t}\right) \cup \cdots \cup f_{n}^{*}\left(w_{0} \ldots w_{t}\right) \\
& =f_{1}^{j}\left(w_{0} \ldots w_{t}\right) \cup \cdots \cup g_{j}\left(w_{0} \ldots w_{t}\right) \cup \cdots \cup f_{n}^{j}\left(w_{0} \ldots w_{t}\right) \\
& ={ }_{i . h .} f_{1}^{j}\left(u_{0} \ldots u_{t}\right) \cup \cdots \cup g_{j}\left(u_{0} \ldots u_{t}\right) \cup \cdots \cup f_{n}^{j}\left(u_{0} \ldots u_{t}\right) \\
& =u_{t+1} .
\end{aligned}
$$

This concludes the proof.

\section{EXPRESSIVENESS, ITERATED BOOLEAN GAMES, AND NASH EQUILIBRIUM}

In this section, we introduce the central concept of this paper: expressiveness in equilibrium. This expressiveness notion is based on the equilibria of iterated Boolean games. We find that some LTLfragments can express properties in equilibrium that they cannot normally express, showing that the two expressiveness notions are to be distinguished. For propositional logic and LTL, however, we show that the two notions are equivalent. Finally, we focus on the next-free fragment $\mathscr{L}_{\mathrm{U}}$, which is known to be the largest stutter-invariant fragment of LTL. We demonstrate and show that the expressive strength in equilibrium of this important fragment lies strictly between that of full LTL and $\mathscr{L}_{U}$ itself.

\subsection{Expressiveness in Equilibrium}

Given a suitable model-theoretic semantics, the expressive power of a logic can be measured in terms of the sets of models it characterises. In the case of temporal propositional logics, the models are infinite sequences of valuations, and by a linear time property we understand any subset $X \subseteq \operatorname{runs}_{\Phi}$ of runs over a set of propositional variables $\Phi$. Thus, we say that an LTL-fragment $\mathscr{L}$ expresses property $X \subseteq$ runs $_{\Phi}$ if there is some formula $\varphi$ in the fragment $\mathscr{L}$ such that $X$ coincides with the set of runs that satisfy $\varphi$, that is, if $X=\operatorname{runs}_{\Phi}(\varphi)$. We also say in this case that $\mathscr{L}$ expresses property $X$ in formulas so as to distinguish the concept from expressiveness in equilibrium to be defined below.

Alternatively, each temporal logic formula over propositional variables $\Phi$ can be seen to define an $\omega$-language over $2^{\Phi}$. Accordingly, every fragment of LTL can also be seen as defining a model of computation, accepting exactly those $\omega$-languages consisting of those $\omega$-words $\rho=v_{0} v_{1} v_{2} \ldots$ over $2^{\Phi}$ for which there is a formula $\varphi$ in $\mathscr{L}$ such that $\rho$ satisfies $\varphi$.

We use the notation $\mathscr{L} \geq \mathscr{L}^{\prime}$ to denote that every property expressible in fragment $\mathscr{L}$ can also be expressed in fragment $\mathscr{L}^{\prime}$. We write $\mathscr{L} \equiv \mathscr{L}^{\prime}$ if both $\mathscr{L} \geq \mathscr{L}^{\prime}$ and $\mathscr{L}^{\prime} \geq \mathscr{L}$, and $\mathscr{L}>\mathscr{L}^{\prime}$ if $\mathscr{L} \geq \mathscr{L}^{\prime}$ but not $\mathscr{L}^{\prime} \geq \mathscr{L}$. We extend these notations in the natural way to models of computation in general, and it is easy to see that the relation $\geq$ is transitive.

In a similar way as do the formulas of a temporal logic, the Nash equilibria of each iterated Boolean game also define a linear time property, namely, the set of runs these equilibria induce. Formally, we say that LTL-fragment $\mathscr{L}$ expresses in equilibrium property $X \subseteq$ runs $s_{\Phi}$, if there is some $\mathscr{L}$-game $G$ such that $X=N E(G)$. Phrased slightly differently, the equilibrium runs of each iterated $\mathscr{L}$-games define an $\omega$-language over $2^{\Phi}$. Accordingly, for each LTL-fragment $\mathscr{L}$, the class of iterated $\mathscr{L}$-games also defines its own model of computation, which we denote by $\mathscr{L}^{N E}$. On this basis of expressible properties, respectively, accepted languages, we can now compare the expressiveness of fragments of linear temporal languages with the expressiveness of the classes of iterated Boolean games based on LTL-fragments.

Intuitively, one might expect that in general for LTL-fragments $\mathscr{L}$, expressiveness in equilibrium is a stronger notion than expressiveness per se, that is, that if $\mathscr{L}$ can express property $X$, it can also express property $X$ in equilibrium. We find that this intuition is generally vindicated, apart from the one notable exception where the set of propositional variables is a singleton $\{p\}$. Then, the empty property $\emptyset$, consisting of no runs whatsoever, is obviously expressed by any unsatisfiable formula 
of $\mathscr{L}$ over $p$, for instance, $p \wedge \neg p$. In this borderline case, however, the empty property cannot be expressed by $\mathscr{L}$ in equilibrium.

To see this, consider an arbitrary $\mathscr{L}$-game over $\Phi=\{p\}$. Then, control over the single variable $p$ can be assigned to a single player $i$ only. Moreover, if the set of equilibrium runs of this game is to be empty, it will also have to be this single player who wants to deviate from every strategy profile. Now, if there is a run satisfying $i$ 's goal, then $i$ does not want to deviate from any strategy profile that underlies it. Consequently, this run is sustained by an equilibrium. If, on the other hand, $i$ 's goal is unsatisfiable, then there is no way she can deviate from any strategy profile to have her goal satisfied. So, in that case, all runs are sustained by a Nash equilibrium. An empty set of equilibrium runs can therefore not be achieved.

In case $\Phi$ contains least two variables, we can construct a two-player $\mathscr{L}$-game with $\operatorname{runs} s_{\Phi}(\varphi)$ as the set of equilibrium runs, for every formula $\varphi$ in an LTL-fragment $\mathscr{L}$. The underlying idea is to assign control over one variable $p$ to the one player and control over another variable $q$ to the other. Then, define the players' preferences such that they both aim to satisfy the formula $\varphi$, but failing that, the first player wants to have the truth-values of $p$ and $q$ to be matched at time $t=0$, whereas the second player wants them to be unmatched. Thus, if a run satisfies $\varphi$, both players are satisfied and neither wants to deviate. If on the other hand, a run does not satisfy $\varphi$ the two players will be caught up in a matching pennies game [Osborne and Rubinstein 1994] on $p$ and $q$, for which there is no (pure) Nash equilibrium. Formally, for any formula $\varphi$ in a fragment $\mathscr{L}$, define the two-player game $G_{\varphi}^{m p}$. Let the two players, $i$ and $j$, such that $i$ controls $p$ and $j$ all other variables, including $q$, that is, $\Phi_{i}=\Phi \backslash\{q\}$ and $\Phi_{j}=\{q\}$. Let furthermore the players' goals be given by:

$$
\gamma_{i}=\varphi \vee(p \leftrightarrow q) \quad \gamma_{j}=\varphi \vee(p \leftrightarrow \bar{q}) .
$$

By showing that $\operatorname{runs}_{\Phi}(\varphi)$ is exactly the set of equilibrium runs of $G_{\varphi}^{m p}$, we obtain the following proposition.

Proposition 4.1. Let $\mathscr{L}$ be an LTL-fragment on $\Phi$ with $|\Phi| \geq 2$. Then, every temporal property that can be expressed by $\mathscr{L}$ in formulas, can also be expressed by $\mathscr{L}$ in equilibrium, that is, $\mathscr{L}^{N E} \geq \mathscr{L}$.

PROOF. Consider an arbitrary property $X$ that is expressed by a formula $\varphi$ in fragment $\mathscr{L}$, and construct the two-player game $G_{\varphi}^{m p}$. It suffices to show that $N E\left(G_{\varphi}^{m p}\right)=\operatorname{runs}_{\Phi}(\varphi)$. To this end, consider an arbitrary run $\rho$ along with a strategy profile $f=\left(f_{i}, f_{j}\right)$ with $\rho=\rho(f)$. If $\rho$ satisfies $\varphi$, both players' goals are satisfied as well. It follows that $f$ is an equilibrium and $\rho \in N E\left(G_{\varphi}^{m p}\right)$. If, on the other hand, $\rho$ does not satisfy $\varphi$, consider $f_{i}(\varepsilon)$ and $f_{j}(\varepsilon)$. If both $p \in f_{i}(\varepsilon)$ and $q \in f_{j}(\varepsilon)$, player $j$ can get his unsatisfied goal satisfied by deviating to any strategy $g_{j}$ with $q \notin g_{j}(\varepsilon)$, as in that case $\rho\left(f_{i}, g_{j}\right) \models p \leftrightarrow \bar{q}$. Player $j$ similarly wants to deviate from $f_{j}$ if $p \notin f_{i}(\varepsilon)$ and $q \notin f_{j}(\varepsilon)$. If it is not the case that $p \in f_{i}(\varepsilon)$ if and only if $q \in f_{j}(\varepsilon)$, it is player $i$ who wants to deviate. In each of these cases it follows that $f=\left(f_{i}, f_{j}\right)$ is not an equilibrium of $G_{\varphi}^{m p}$, as desired.

Since we have established that in virtually all cases, properties that can be expressed by a fragment $\mathscr{L}$ in formulas can also expressed by $\mathscr{L}$ in equilibrium, one may wonder if the inverse statement holds as well. The game Boolean Ballet, as presented informally in Example 1.1, shows exactly that this is not generally the case: there exist LTL-fragments that cannot express certain properties that they can express in equilibrium. This is in particular the case for the fragment $\mathscr{L}_{\mathrm{F}, \mathrm{G}}$, which, as a sublogic LTL-fragment $\mathscr{L}_{\mathrm{U}}$, is known to be stutter-invariant. In the proof of this result we buttress our informal reasoning in the introduction by a formally precise argument.

PROPOSITION 4.2. Let $\Phi$ be a set of propositional variables with $|\Phi| \geq 2$. Then, it is not generally the case for every $L T L$-fragment on $\Phi$ that every temporal property that can be expressed by $\mathscr{L}$ in equilibrium, can also be expressed by $\mathscr{L}$ in formulas. In particular, this does not hold for the LTL-fragment $\mathscr{L}_{\mathrm{F}, \mathrm{G}}$, and, hence, $\mathscr{L}_{\mathrm{F}, \mathrm{G}}^{N E}>\mathscr{L}_{\mathrm{F}, \mathrm{G}}$. 
Proof. As we may assume $|\Phi| \geq 2$, by Proposition 4.1 we immediately have that every property that $\mathscr{L}_{\mathrm{F}, \mathrm{G}}$ can express, $\mathscr{L}_{\mathrm{F}, \mathrm{G}}$ can also express in equilibrium. To show that there is a property that $\mathscr{L}_{\mathrm{F}, \mathrm{G}}^{N E}$ can express, but $\mathscr{L}_{\mathrm{F}, \mathrm{G}}$ cannot, consider the two-person iterated Boolean game $G_{b b}$ referred to as "Boolean Ballet" in Example 1.1 in the introduction. (For expositional convenience, we prove the statement for the case where $\Phi=\{p, q\}$. The argument, however, can be easily extended to the more general case where $\{p, q\} \subseteq \Phi$.) Recall from Example 3.1 that

$$
\begin{aligned}
\Phi_{i} & =\{p\} & \Phi_{j} & =\{q\} \\
\gamma_{i} & =\mathrm{G}(p \leftrightarrow q) & \gamma_{j} & =\mathrm{F} p q \wedge \mathrm{F} p \bar{q} .
\end{aligned}
$$

We show that for every run $\rho=v_{0} v_{1} v_{2} \ldots$, we have that $\rho \in N E\left(G_{b b}\right)$ if and only if both $v_{t}=p q$ or $v_{t}=\bar{p} \bar{q}$ for all $t \geq 0$, and $v_{s}=p q$ for at most one $s \geq 0$. As the Nash equilibria thus define a temporal property that is not stutter-invariant—observe that $p q \bar{p} \bar{q} \bar{p} \bar{q} \bar{p} \bar{q} \ldots$ is an equilibrium, but $p q p q \bar{p} \bar{q} \bar{p} \bar{q} \bar{p} \bar{q} \ldots$ is not-this suffices for a proof.

To this end, first consider an arbitrary run $\rho=v_{0} v_{1} v_{2} \ldots$ such that $v_{t}=p q$ or $v_{t}=\bar{p} \bar{q}$ for all $t \geq 0$ and $v_{s}=p q$ for at most one $s \geq 0$. Also consider the naive strategies $f_{i}$ and $f_{j}$ defined such that, for every $t \geq 0$ and history $w_{0} \ldots w_{t-1}$,

$$
f_{i}\left(w_{0} \ldots w_{t}\right)=v_{t+1} \cap \Phi_{i} \quad \text { and } \quad f_{j}\left(w_{0} \ldots w_{t}\right)=v_{t+1} \cap \Phi_{j},
$$

(on the understanding that $w_{0} \ldots w_{t-1}=\varepsilon$ for $t=0$ ). It is then easily appreciated that $\rho\left(f_{i}, f_{j}\right)=$ $v_{0} v_{i} v_{j} \ldots$. To see that $f=\left(f_{i}, f_{j}\right)$ is a Nash equilibrium, first observe that $\rho(f) \models \gamma_{i}$. Thus player $i$ has her goal satisfied and she does not want to deviate. For player $j$, however, $\rho(f) \forall \gamma_{j}$. Now, assume for contradiction that there is a strategy $g_{j}$ such that $\rho\left(f_{i}, g_{j}\right) \models \gamma_{j}$, that is, that player $j$ would like to deviate from $f$ and play $g_{j}$. Let $\rho\left(f_{i}, g_{j}\right)=v_{0}^{\prime} v_{1}^{\prime} v_{2}^{\prime}, \ldots$ Then, a $t^{*} \geq 0$ exists such that $v_{t^{*}}^{\prime}=p \bar{q}$. Moreover, as we defined $f_{i}$ as a naive strategy, $v_{t^{*}}=p q$ and $v_{t}=\bar{p} \bar{q}$ for for all $t \neq t^{*}$. Again because $f_{i}$ is naive, $v_{t}^{\prime}=\bar{p} q$ or $v_{t}^{\prime}=\bar{p} \bar{q}$ for all $t \neq t^{*}$. It follows that $\rho\left(f_{i}, g_{j}\right) \not \neq \mathrm{F} p \bar{q}$ and, hence, $\rho\left(f_{i}, g_{j}\right) \not \forall \gamma_{j}$, a contradiction.

For the opposite direction, assume that $f=\left(f_{i}, f_{j}\right)$ is a strategy profile such that $\rho\left(f_{i}, f_{j}\right)=$ $v_{0} v_{i} v_{j} \ldots$, and, for contraposition, that either $(i)$ it is not the case that $v_{t}=p q$ or $v_{t}=\bar{p} \bar{q}$ for all $t \geq 0$, or $(i i)$ there are $t^{\prime \prime}>t^{\prime} \geq 0$ with $v_{t^{\prime}}=v_{t^{\prime \prime}}=p q$. We show that $f$ is not a Nash equilibrium.

If $(i)$, observe that $\rho\left(f_{i}, f_{j}\right) \not \forall \gamma_{i}$. Now, define strategy $g_{i}$ such that for all $t \geq 0$ and histories $w_{0} \ldots w_{t-1}$,

$$
g_{i}\left(w_{0} \ldots w_{t-1}\right)= \begin{cases}p & \text { if } f_{j}\left(w_{0} \ldots w_{t-1}\right)=q, \\ \bar{p} & \text { if } f_{j}\left(w_{0} \ldots w_{t-1}\right)=\bar{q}\end{cases}
$$

(again on the understanding that $w_{0} \ldots w_{t-1}=\varepsilon$ for $t=0$ ). Intuitively, $g_{i}$ inspects strategy $f_{j}$ to predict at each time $t$ to what truth-value player $j$ sets $q$, and matches $p$ 's truth-value accordingly. Let $\rho\left(g_{i}, f_{j}\right)=w_{0}^{\prime} w_{1}^{\prime} w_{2}^{\prime} \ldots$. By means of a straightforward inductive argument, which we here omit, it can then be shown that $w_{t}^{\prime}=p q$ or $w_{t}^{\prime}=\bar{p} \bar{q}$ for all $t \geq 0$, and hence $\rho\left(g_{i}, f_{j}\right) \models \gamma_{i}$. Accordingly, $f$ is not a Nash equilibrium.

If $(i i)$, observe that we may assume that $v_{t}=p q$ or $v_{t}=\bar{p} \bar{q}$ for all $t \geq 0$. Then, obviously, $\rho\left(f_{i}, f_{j}\right) \not \forall \gamma_{j}$. Now define $g_{j}$ such that for all $t \geq 0$ and histories $w_{0}, \ldots, w_{t-1}$ (on the understanding that $w_{0} \ldots w_{t-1}=\varepsilon$ for $\left.t=0\right)$ :

$$
g_{j}\left(w_{0} \ldots w_{t-1}\right)= \begin{cases}f_{j}\left(w_{0} \ldots w_{t-1}\right) & \text { if } t \leq t^{\prime \prime}, \\ \bar{q} & \text { otherwise. }\end{cases}
$$

Thus, by using strategy $g_{j}$, player $j$ makes the same choices as with $f_{j}$ up to time $t^{\prime \prime}-1$, where it deviates by choosing $\bar{q}$ instead of $q$. Let $\rho\left(f_{i}, g_{j}\right)=w_{0}^{\prime \prime} w_{i}^{\prime \prime} w_{j}^{\prime \prime}, \ldots$ By means of another straightforward inductive argument, which again we omit, it can then be show that $w_{0}^{\prime \prime} \ldots w_{t^{\prime \prime}-1}^{\prime \prime}=v_{0} \ldots v_{t^{\prime \prime}-1}$. In particular, it holds that $w_{t^{\prime}}^{\prime \prime}=p q$. For $t^{\prime \prime}$, moreover, we have both

$$
f_{i}\left(w_{0}^{\prime \prime} \ldots w_{t^{\prime \prime}-1}^{\prime \prime}\right)=f_{i}\left(v_{0} \ldots v_{t^{\prime \prime}-1}\right)=p \quad \text { and } \quad g_{j}\left(w_{0}^{\prime \prime} \ldots w_{t^{\prime \prime}-1}^{\prime \prime}\right)=\bar{q}
$$


that is, $w_{t^{\prime \prime}}^{\prime \prime}=p \bar{q}$. Accordingly, $\rho\left(f_{j}, g_{j}\right) \models \gamma_{j}$. Hence, as player $j$ can thus deviate from $f$ by playing $g_{j}$ and satisfy his goal, we may conclude that $f$ is not a Nash equilibrium, as desired.

We conclude this section with the easy but useful observation concerning the relative expressive power of different fragments: if a fragment $\mathscr{L}_{1}$ is at least as expressive as $\mathscr{L}_{2}$ in formulas, then $\mathscr{L}_{1}$ is also at least as expressive as $\mathscr{L}_{2}$ in equilibrium.

Proposition 4.3. For fragments $\mathscr{L}_{1}$ and $\mathscr{L}_{2}$, we have $\mathscr{L}_{1} \geq \mathscr{L}_{2}$ implies $\mathscr{L}_{1}^{N E} \geq \mathscr{L}_{2}^{N E}$.

PROOF. Assume $\mathscr{L}_{1} \geq \mathscr{L}_{2}$ and consider an arbitrary temporal property $X$ such that $X=$ $N E\left(G_{2}\right)$ for some $\mathscr{L}_{2}$-game $G_{2}=\left(N, \Phi, \Phi_{1}, \ldots, \Phi_{n}, \gamma_{1}, \ldots, \gamma_{n}\right)$ with $\gamma_{i} \in \mathscr{L}_{1}$ for all players $i$. Then, for each player $i$ there is an formula $\gamma_{i}^{\prime} \in \mathscr{L}_{1}$ such that $\operatorname{runs}_{\Phi}\left(\gamma_{i}\right)=\operatorname{runs}_{\Phi}\left(\gamma_{i}^{\prime}\right)$. Let $G_{1}=$ $\left(N, \Phi, \Phi_{1}, \ldots, \Phi_{n}, \gamma_{1}^{\prime}, \ldots, \gamma_{n}^{\prime}\right)$. Note that the strategies for each player in $G_{1}$ and $G_{2}$ are identical. Then, clearly, $N E\left(G_{1}\right)=N E\left(G_{2}\right)=X$. Consider the following equivalences for every $\rho \in \operatorname{runs}_{\Phi}$.

$$
\begin{aligned}
& \rho \in N E\left(G_{1}\right) \\
& \text { iff } \rho=\rho\left(f^{*}\right) \text { and } f^{*} \text { a Nash equilibrium of } G_{1} \\
& \text { iff } \rho=\rho\left(f^{*}\right) \text { and } \rho\left(f_{-i}^{*}, f_{i}^{\prime}\right) \models \gamma_{i} \text { implies } \rho\left(f^{*}\right) \models \gamma_{i}, \text { for all players } i \text { and strategies } f_{i} \\
& \text { iff } \rho=\rho\left(f^{*}\right) \text { and } \rho\left(f_{-i}^{*}, f_{i}^{\prime}\right) \models \gamma_{i}^{\prime} \text { implies } \rho\left(f^{*}\right) \models \gamma_{i}^{\prime} \text {, for all players } i \text { and strategies } f_{i} \\
& \text { iff } \rho=\rho\left(f^{*}\right) \text { and } f^{*} \text { a Nash equilibrium of } G_{2} \\
& \text { iff } \rho \in N E\left(G_{2}\right) \text {. }
\end{aligned}
$$

This concludes the proof.

\subsection{Propositional Logic: The Empty Fragment $\mathscr{L}_{\varnothing}$}

In the previous section we saw that an LTL-fragment can be more expressive in equilibrium than it is in formulas. This, however, is not necessarily the case. A prime example is propositional logic, that is, the empty LTL-fragment $\mathscr{L}_{\varnothing}$ with no temporal operators. A fundamental observation in the literature on Boolean games is that for any given Boolean game there is a formula characterising the set of Nash equilibrium outcomes. More formally, given an $\mathscr{L}_{\varnothing}$-game $\left(N, \Phi, \Phi_{1}, \ldots, \Phi_{n}, \gamma_{1}, \ldots, \gamma_{n}\right)$, the set of equilibrium runs is characterised by

$$
\eta(G)=\bigwedge_{i \in N}\left(\bigvee_{\theta: \Phi_{i} \rightarrow\{T, \perp\}} \theta\left(\gamma_{i}\right) \rightarrow \gamma_{i}\right),
$$

where $\theta\left(\gamma_{i}\right)$ results from $\gamma_{i}$ by replacing every occurrence of a propositional variable $p$ in $\Phi_{i}$ by the Boolean T or $\perp$ as specified by $\theta(p)$ [Bonzon et al. 2006]. As an immediate consequence, we find that $\mathscr{L}_{\varnothing}$ can express every property that it can express in equilibria, and-provided that $\Phi$ contains at least two propositional variables-vice versa.

Proposition 4.4. Let $\mathscr{L}$ be an LTL-fragment on $\Phi$ with $|\Phi| \geq 2$. Then, $\mathscr{L}_{\varnothing}$ can express property $X$ in formulas if and only if $\mathscr{L}_{\varnothing}$ can express $X$ in equilibrium, that is, $\mathscr{L}_{\varnothing} \equiv \mathscr{L}_{\varnothing}^{N E}$.

ProOF. The "only if"-direction is immediate by Proposition 4.1. For the "if"-direction, let $X$ be an arbitrary temporal property that can be expressed by $\mathscr{L}_{\varnothing}$ in equilibrium. Then, there is an $\mathscr{L}_{\phi^{-}}$ game $G$ with $X=N E(G)$. Dealing with $\mathscr{L}_{\varnothing}$, it is then well known that $X=\operatorname{runs}_{\Phi}(\eta(G))$. Conclude by observing that $\eta(G)$ is a formula of $\mathscr{L}_{\varnothing}$.

It may be worth observing that proof of Proposition 4.4 shows something in addition to the equiexpressiveness of propositional logic in formulas and in equilibrium: the formula scheme $\eta(G)$ provides a uniform way to find a propositional formula characterising the equilibrium runs of a Boolean game.

\subsection{LTL: The Full Fragment}

We now turn to the question how expressive LTL is with respect to $\operatorname{LTL}^{N E}$, that is, to what extent the temporal properties defined by the Nash equilibria of LTL-games can be expressed in formulas 
by LTL itself. Again under the assumption that there be at least two propositional variables, we show that LTL is just as expressive as it is in equilibrium, that is, LTL $\equiv$ LTL $^{N E}$ (see, Theorem 4.8, below).

Key to this issue are those temporal properties (or, more generally, $\omega$-languages) that are noncounting [Mateescu and Salomaa 1997; Strejček 2004]. Formally, and adapted to our setting, a temporal property $X$ over $2^{\Phi}$ is non-counting if there is an index $n_{0}>0$ such that for all $k \geq n_{0}$ and histories $\pi, \pi_{0}, \pi_{1}$, and $\pi_{2}$, the following equivalences hold:

(i) $\quad \pi_{0} \pi^{k} \pi_{1} \pi_{2}^{\omega} \in X \quad$ if and only if $\pi_{0} \pi^{k+1} \pi_{1} \pi_{2}^{\omega} \in X$, and

(ii) $\pi_{0}\left(\pi_{1} \pi^{k} \pi_{2}\right)^{\omega} \in X \quad$ if and only if $\pi_{0}\left(\pi_{1} \pi^{k+1} \pi_{2}\right)^{\omega} \in X$.

Intuitively, the property of being non-counting can be seen as a weak form of stutter-invariance, where a string can only be 'stuttered' after it has been stuttered a large enough number of times already. As a prime example of a counting property over runs $s_{\Phi}$ consider the one consisting of all runs $v_{0} v_{1} v_{2} \ldots$ for which there is an odd $t \geq 0$ such that $v_{t^{\prime}}=\{p\}$ for all $t^{\prime} \leq t$ and $v_{t^{\prime \prime}}=\{q\}$ for all $t^{\prime \prime}>t$, that is the property defined by the $\omega$-regular expression $(\{p\} ;\{p\})^{*} ;\{q\}^{\omega}$. Whereas for every even $k \geq 0$ we have that the run $\{p\}^{k}\{q\}^{\omega}$ belongs to this property, the run $\{p\}^{k+1}\{q\}^{\omega}$ does not.

[Kučera and Strejček 2005] have characterised the LTL-properties as those that are both $\omega$-regular and non-counting.

THEOREM 4.5 ([KUČERA AND STREJČEK 2005]). A property $X \subseteq$ runs $\Phi$ can be expressed by LTL if and only if $X$ is $\omega$-regular and non-counting.

In the remainder of this section, we therefore demonstrate that every LTL-game $G$ the set $N E(G)$ of equilibrium runs is both non-counting and $\omega$-regular, showing the former first.

PROPOSITION 4.6. For every LTL-game $G$, the set $N E(G)$ of equilibrium runs is non-counting.

Proof. Consider an arbitrary LTL-game $G=\left(N, \Phi, \Phi_{1}, \ldots, \Phi_{n}, \gamma_{1}, \ldots, \gamma_{n}\right)$. As the goal $\gamma_{i}$ of each player $i$ as well as its negation $\neg \gamma_{i}$ define LTL-properties, by Theorem 4.5, there are indices $n_{\gamma_{i}}, n_{\neg \gamma_{i}} \geq 0$ in virtue of which, respectively, $\operatorname{runs}_{\Phi}\left(\gamma_{i}\right)$ and $\operatorname{runs_{\Phi }}\left(\neg \gamma_{i}\right)$ are non-counting properties. Now let

$$
n_{0}=1+\max \left\{n_{\gamma_{i}}, n_{\neg \gamma_{i}}: i \in N\right\} .
$$

Thus for every player $i$ we have $n_{\gamma_{i}}, n_{\neg \gamma_{i}}<n_{0}$, that is, the index $n_{0}$ is chosen sufficiently large so as to apply to all the player's goals and their negations. To show that the set $N E(G)$ is non-counting with respect to index $n_{0}$, we consider an arbitrary $k \geq n_{0}$, and arbitrary histories $\pi, \pi_{0}, \pi_{1}$, and $\pi_{2}$, and show that both:

(i) $\quad \pi_{0} \pi^{k} \pi_{1} \pi_{2}^{\omega} \in N E(G) \quad$ if and only if $\pi_{0} \pi^{k+1} \pi_{1} \pi_{2}^{\omega} \in N E(G)$,

(ii) $\pi_{0}\left(\pi_{1} \pi^{k} \pi_{2}\right)^{\omega} \in N E(G)$ if and only if $\pi_{0}\left(\pi_{1} \pi^{k+1} \pi_{2}\right)^{\omega} \in N E(G)$.

Thus, we have to provide proofs for four statements - the "if"-direction and "only if"-direction of both $(i)$ and $(i i)$. Each of which has a similar structure.

For the "only if"-direction of $(i)$, let $\rho=\pi_{0} \pi^{k} \pi_{1} \pi_{2}^{\omega}$ and $\rho^{\prime}=\pi_{0} \pi^{k+1} \pi_{1} \pi_{2}^{\omega}$. As $\gamma_{i}$ defines a non-counting property for every player $i$, by choice of $n_{0}$, we have $\rho \models \gamma_{i}$ if and only if $\rho^{\prime} \models \gamma_{i}$ for every player $i$. Assume that there is a Nash equilibrium $f^{*}=\left(f_{1}^{*}, \ldots, f_{n}^{*}\right)$ that sustains $\rho$, that is $\rho\left(f^{*}\right)=\pi_{0} \pi^{k} \pi_{1} \pi_{2}^{\omega}$. On this basis we construct another Nash equilibrium $g^{*}=\left(g_{1}^{*}, \ldots, g_{n}^{*}\right)$ of $G$ that sustains $\rho^{\prime}$, that is, $\rho\left(g^{*}\right)=\pi_{0} \pi^{k+1} \pi_{1} \pi_{2}^{\omega}$, and $\rho\left(g^{*}\right) \in N E(G)$.

To this end, first observe that every history $\eta$ is of the form $\pi_{c o m} \pi_{d e v}$, where $\pi_{c o m}$ is the unique maximal common prefix of $\rho^{\prime}$ and $\eta$, and $\pi_{d e v}$ is a tail piece of $\eta$ that 'deviates' from $\rho^{\prime}$. Note that both $\pi_{c o m}$ and $\pi_{d e v}$ may be the empty sequence $\varepsilon$. For every history $\eta=\pi_{c o m} \pi_{d e v}$ there are then two possibilities: $\pi_{\text {com }}<\pi_{0} \pi^{k}$, or $\pi_{\text {com }}=\pi_{0} \pi^{k} \pi^{\prime}$ for some $\pi^{\prime} \leq \pi \pi_{1} \pi_{2}^{\omega}$. That is, $\eta$ differs (if at all) from $\rho^{\prime}$ either before the $k$ th iteration of $\pi$ in $\rho^{\prime}$ is completed, or thereafter. Observe that 
$\pi_{\text {com }}$ may be the empty sequence. Now define $g^{*}=\left(g_{1}^{*}, \ldots, g_{n}^{*}\right)$ such that, for each player $i$ and history $\eta=\pi_{c o m} \pi_{d e v}$,

$$
g_{i}^{*}(\eta)= \begin{cases}f_{i}^{*}\left(\pi_{c o m} \pi_{d e v}\right) & \text { if } \pi_{c o m}<\pi_{0} \pi^{k} \\ f_{i}^{*}\left(\pi_{0} \pi^{k-1} \pi^{\prime} \pi_{d e v}\right) & \text { if } \pi_{c o m}=\pi_{0} \pi^{k} \pi^{\prime} \text { for some } \pi^{\prime} \leq \pi \pi_{1} \pi_{2}^{\omega} .\end{cases}
$$

Observe that $g_{i}^{*}$ is well-defined for each player $i$ because we may assume that $k \geq 1$. Intuitively, the strategy profiles $f^{*}$ and $g^{*}$ are the same apart that by playing $g^{*}$ the players repeat the $k$ th iteration of $\pi$. Observe that this also includes the behaviour of $g_{i}^{*}$ on histories that are not a prefix of the run $\rho^{\prime}=\pi_{0} \pi^{k+1} \pi_{1} \pi_{2}^{\omega}$. By means of an inductive argument we defer to the appendix, it can then be shown that $\rho\left(g^{*}\right)=\pi_{0} \pi^{k+1} \pi_{1} \pi_{2}^{\omega}$.

It remains to be shown that $g^{*}=\left(g_{1}^{*}, \ldots, g_{n}^{*}\right)$ is also a Nash equilibrium. To this end, assume for contradiction that there is a strategy $g_{j}^{\prime}$ for some player $j$ such that $\rho\left(g^{*}\right) \not \models \gamma_{j}$ and $\rho\left(g_{-j}^{*}, g_{j}^{\prime}\right) \models \gamma_{i}$. Recall that $\gamma_{j}$ defines a non-counting property for index $n_{0}$. As $\rho\left(f^{*}\right)=\pi_{0} \pi^{k} \pi_{1} \pi_{2}^{\omega}$ and $\rho\left(g^{*}\right)=$ $\pi_{0} \pi^{k+1} \pi_{1} \pi_{2}^{\omega}$, we thus obtain that $\rho\left(f^{*}\right) \not \models \gamma_{j}$.

To see that player $j$ also wants to deviate from $f^{*}$, let $\rho\left(g_{-j}^{*}, g_{j}^{\prime}\right)=\pi_{\text {com }}^{\prime} \rho_{\text {dev }}^{\prime}$, where $\pi_{\text {com }}^{\prime}$ is the maximal common prefix of $\rho\left(g^{*}\right)$ and $\rho\left(g_{-j}^{*}, g_{j}^{\prime}\right)$, and $\rho_{c o m}^{\prime}$ a 'deviant' continuation of the run. Observe that $\pi_{\text {com }}^{\prime}$ is finite because $\rho\left(g^{*}\right) \neq \rho\left(g_{-j}^{*}, g_{j}^{\prime}\right)$. We distinguish two cases: either $\pi_{c o m}^{\prime}<\pi_{0} \pi^{k}$ or $\pi_{\text {com }}^{\prime}=\pi_{0} \pi^{k} \pi^{\prime}$ for some $\pi^{\prime} \leq \pi \pi_{1} \pi_{2}^{\omega}$, that is, by playing $g_{j}^{\prime}$ player $j$ deviates from $g^{*}$ either before or after the $k$ th iteration of $\pi$ is completed.

If the former, we find that $\rho\left(f_{-j}^{*}, g_{j}^{\prime}\right)=\rho\left(g_{-j}^{*}, g_{j}^{\prime}\right)$, and hence immediately $\rho\left(f_{-j}^{*}, g_{j}^{\prime}\right) \models \gamma_{j}$.

If the latter, $\rho\left(g_{-j}^{*}, g_{j}^{\prime}\right)=\pi_{0} \pi^{k} \pi^{\prime} \rho_{d e v}$ for some $\pi^{\prime} \leq \pi \pi_{1} \pi_{2}^{\omega}$ and some 'deviant' run $\rho_{d e v}$. We define a strategy $f_{j}^{\prime}$ for player $j$ such that $\rho\left(f_{-j}^{*}, f_{j}^{\prime}\right)=\pi_{0} \pi^{k-1} \pi^{\prime} \rho_{d e v}$. To this end, first observe that every history is of the form $\eta=\pi_{c o m}^{\prime \prime} \pi_{d e v}^{\prime \prime}$, where $\pi_{c o m}^{\prime \prime}$ is the maximal common prefix of $\eta$ and $\pi_{0} \pi^{k-1} \pi^{\prime} \rho_{d e v}$, and $\pi_{d e v}^{\prime \prime}$ some 'deviant' history. Now define strategy $f_{j}^{\prime}$ for player $j$ such that, for every $\eta=\pi_{c o m}^{\prime \prime} \pi_{d e v}^{\prime \prime}$,

$$
f_{j}^{\prime}\left(\pi_{c o m}^{\prime \prime} \pi_{d e v}^{\prime \prime}\right)= \begin{cases}g_{j}^{\prime}\left(\pi_{c o m}^{\prime \prime} \pi_{d e v}^{\prime \prime}\right) & \text { if } \pi_{c o m}^{\prime \prime}<\pi_{0} \pi^{k-1} \\ g_{j}^{\prime}\left(\pi_{0} \pi^{k} \pi^{\prime} \pi_{d e v}^{\prime \prime}\right) & \text { if } \pi_{c o m}^{\prime \prime}=\pi_{0} \pi^{k-1} \pi^{\prime} \text { with } \pi^{\prime} \leq \pi \pi_{1} \pi_{2}^{\omega} .\end{cases}
$$

It can then be shown by an inductive argument here omitted (but see the appendix) that $\rho\left(f_{-j}^{*}, f_{j}^{\prime}\right)=$ $\pi_{0} \pi^{k-1} \pi^{\prime} \rho_{d e v}$. As, by definition of $n_{0}$ we have $k-1 \geq n_{\gamma_{j}}$, and, moreover, $\pi_{0} \pi^{k} \pi^{\prime} \rho_{d e v} \models \gamma_{j}$, it would follow that also $\rho\left(f_{-j}^{*}, f_{j}^{\prime}\right) \models \gamma_{j}$.

In either case, it would follow that $f^{*}$ is not an equilibrium, a contradiction, as desired.

The "if"-direction of $(i)$ has basically the same structure as the "only if"-direction. Let $f^{*}=$ $\left(f_{1}^{*}, \ldots, f_{n}^{*}\right)$ be a Nash equilibrium of $G$ with $\rho\left(f^{*}\right)=\pi_{0} \pi^{k+1} \pi_{1} \pi_{2}^{\omega} \in N E(G)$. We define strategy profile $g^{*}=\left(g_{1}^{*}, \ldots, g_{n}^{*}\right)$ such that $\rho\left(g^{*}\right)=\pi_{0} \pi^{k} \pi_{1} \pi_{2}^{\omega}$ and show that $g^{*}$ is a Nash equilibrium of $G$ as well.

Observe that every history $\eta$ is of the form $\pi_{c o m} \pi_{d e v}$, where $\pi_{c o m}$ is the largest common prefix of $\pi_{0} \pi^{k} \pi_{1} \pi_{2}^{\omega}$. Then, define, for every player $i$ and every history $\eta=\pi$,

$$
g_{i}^{*}\left(\pi_{c o m} \pi_{d e v}\right)= \begin{cases}f^{*}\left(\pi_{c o m} \pi_{d e v}\right) & \text { if } \pi_{c o m}<\pi_{0} \pi^{k}, \\ f^{*}\left(\pi_{0} \pi^{k} \pi \pi_{d e v}^{\prime}\right) & \text { if } \pi_{c o m}=\pi_{0} \pi^{k} \pi^{\prime} \text { for some } \pi^{\prime} \leq \pi_{1} \pi_{2}^{\omega} .\end{cases}
$$

By means of an inductive argument here omitted it can then be proven that $\rho\left(g^{*}\right)=\pi_{0} \pi^{k} \pi_{1} \pi_{2}^{*}$. To demonstrate that $g^{*}$ is also a Nash equilibrium, assume that $g^{*}$ is not. Accordingly, there is some player $j$ with $\rho\left(g^{*}\right) \not \models \gamma_{j}$ and some strategy $g_{j}^{\prime}$ for $j$ such that $\rho\left(g_{-j}^{*}, g_{j}^{\prime}\right) \models \gamma_{j}$. As $\neg \gamma_{i}$ is a noncounting property with an index not larger than $k$, it follows that $\rho\left(f^{*}\right) \forall \forall \gamma_{j}$. 
If, by playing $g_{j}^{\prime}$, player $j$ unilaterally deviates from $g^{*}$ before the $k$-th iteration of $\pi$ is completed-that is, if $\pi_{\text {com }}^{\prime}<\pi_{0} \pi^{k}$, where $\pi_{\text {com }}^{\prime}$ is the maximal common prefix of $\rho\left(g^{*}\right)$ and $\rho\left(g_{-j}^{*}, g_{j}^{\prime}\right)$-it can be shown by an inductive argument here omitted that $\rho\left(f_{-j}^{*}, g_{j}^{\prime}\right)=\rho\left(g_{-j}^{*}, g_{j}^{\prime}\right)$. Hence, $\rho\left(f_{-j}^{*}, g_{j}^{\prime}\right) \models \gamma_{j}$. It would thus follow that $f^{*}$ were not a Nash equilibrium, a contradiction.

Now assume that, by playing $g_{j}^{\prime}$, player $j$ unilaterally deviates from $g^{*}$ after the $k$-th iteration of $\pi$ is completed-that is, that $\pi_{\text {com }}^{\prime} \geq \pi_{0} \pi^{k}$, where $\pi_{c o m}^{\prime}$ is the maximal common prefix of $\rho(g *)$ and $\rho\left(g_{-j}^{*}, g_{j}^{\prime}\right)$. Then $\rho\left(g_{-j}^{*}, g_{j}^{\prime}\right)=\pi_{0} \pi^{k} \pi^{\prime \prime} \rho_{d e v}$, where $\pi^{\prime \prime} \leq \pi_{1} \pi_{2}^{\omega}$. We now define strategy $f_{j}^{\prime}$ such that for every history $\eta$ of the form $\pi_{c o m}^{\prime \prime} \pi_{d e v}^{\prime \prime}$ where $\pi_{c o m}^{\prime \prime}$ is the maximal common prefix of $\eta$ and $\pi_{0} \pi^{k+1} \pi_{1} \pi_{2}^{\omega}$,

$$
f_{j}^{\prime}\left(\pi_{\text {com }}^{\prime \prime} \pi_{\text {dev }}^{\prime \prime}\right)= \begin{cases}g_{j}^{\prime}\left(\pi_{\text {com }}^{\prime \prime} \pi_{\text {dev }}^{\prime \prime}\right) & \text { if } \pi_{\text {com }}^{\prime \prime}<\pi_{0} \pi, \\ g_{j}^{\prime}\left(\pi_{0}, \pi^{\prime}, \pi_{\text {dev }}^{\prime \prime}\right) & \text { if } \pi_{\text {com }}^{\prime \prime}=\pi_{0} \pi \pi^{\prime} \text { where } \pi^{\prime} \leq \pi^{k} \pi_{1} \pi_{2}^{\omega} .\end{cases}
$$

Defined thus, we find by an inductive argument omitted here that $\rho\left(f_{-j}^{*}, f_{j}^{\prime}\right)=\pi_{0} \pi^{k+1} \pi^{\prime \prime} \rho_{d e v}$. As $k>n_{\gamma_{j}}$, it follows that $\rho\left(f_{-j}^{*}, f_{j}^{\prime}\right) \models \gamma_{j}$, and again we may conclude that $f^{*}$ is not a Nash equilibrium, a contradiction.

For the "only if"-direction of $(i i)$, assume that $f^{*}=\left(f_{1}^{*}, \ldots, f_{n}^{*}\right)$ is an equilibrium with $\rho\left(f^{*}\right)=$ $\pi_{0}\left(\pi_{1} \pi^{k} \pi_{2}\right)^{\omega}$. We define profile $g^{*}=\left(g_{1}^{*}, \ldots, g_{n}^{*}\right)$ such that $\rho\left(g^{*}\right)=\pi_{0}\left(\pi_{1} \pi^{k+1} \pi_{2}\right)^{\omega}$, and show that $g^{*}$ is an equilibrium as well. In order to define $g^{*}$, first observe that every history is of the form $\eta=\pi_{c o m} \pi_{d e v}$, where $\pi_{c o m}$ is the maximal common prefix of $\eta$ and $\pi_{0}\left(\pi_{1} \pi^{k+1} \pi_{2}\right)^{\omega}$, and $\pi_{d e v}$ a "deviant" continuation. Moreover, either $\pi_{\text {com }}<\pi_{0}$ or $\pi_{c o m}=\pi_{0}\left(\pi_{1} \pi^{k+1} \pi_{2}\right)^{\ell} \pi^{\prime}$ for $\ell \geq 0$ and some $\pi^{\prime}<\pi_{1} \pi^{k+1} \pi_{2}$. Given $\eta=\pi_{c o m} \pi_{d e v}$, define $\check{\pi}_{c o m}$ such that $\check{\pi}_{c o m}=\pi_{c o m}$ if the former and

$$
\check{\pi}_{\text {com }}= \begin{cases}\pi_{0}\left(\pi_{1} \pi^{k} \pi_{2}\right)^{\ell} \pi^{\prime} & \text { if } \pi^{\prime}<\pi_{1} \pi^{k} \\ \pi_{0}\left(\pi_{1} \pi^{k} \pi_{2}\right)^{\ell} \pi_{1} \pi^{k-1} \pi^{\prime \prime} & \text { if } \pi^{\prime}=\pi_{1} \pi^{k} \pi^{\prime \prime} \text { for some } \pi^{\prime \prime} \leq \pi \pi_{2},\end{cases}
$$

if the latter. Subsequently, define for every player $i$ and history $\eta=\pi_{c o m} \pi_{d e v}$,

$$
g_{i}^{*}\left(\pi_{c o m} \pi_{d e v}\right)=f_{i}^{*}\left(\check{\pi}_{c o m} \pi_{d e v}\right) .
$$

Defined thus, $\rho\left(g^{*}\right)=\pi_{0}\left(\pi_{1} \pi^{k+1} \pi_{2}\right)^{\omega}$.

Now assume for a contradiction that $g^{*}$ is not a Nash equilibrium. Then, there is a player $j$ with $\rho\left(g^{*}\right) \not \models \gamma_{j}$ and some strategy $g_{j}^{\prime}$ for $j$ such that $\rho\left(g_{-j}^{*}, g_{j}^{\prime}\right) \models \gamma_{j}$. As $n_{\neg \gamma_{j}}<k$, then also $\rho\left(f^{*}\right) \not \forall \gamma_{j}$.

Let $\rho\left(g_{-j}^{*}, g_{j}^{\prime}\right)=\pi_{c o m}^{\prime} \rho_{d e v}^{\prime}$, where $\pi_{\text {com }}^{\prime}$ is the maximum common prefix of $\rho\left(g^{*}\right)$ and $\rho\left(g_{-j}^{*}, g_{j}^{\prime}\right)$, and $\rho_{d e v}^{\prime}$ a 'deviant' continuation.

If $\pi_{\text {com }}^{\prime}<\pi_{0} \pi_{1} \pi^{k}$, then $\rho\left(g_{-j}^{*}, g_{j}^{\prime}\right)=\rho\left(f_{-j}^{*}, g_{j}^{\prime}\right)$. Hence, $\rho\left(f_{-j}, g_{j}^{\prime}\right) \not \forall \gamma_{j}$, which would signify that $f^{*}$ were not a Nash equilibrium, a contradiction. If, on the other hand, $\pi_{c o m}^{\prime}=\pi_{0}\left(\pi_{1} \pi^{k+1} \pi_{2}\right)^{m} \pi^{\prime}$ for some $m \geq 0$ and $\pi \leq \pi_{1} \pi^{k+1} \pi_{2}$. Then, $\rho\left(g_{-j}^{*}, g_{j}^{\prime}\right)=\pi_{0}\left(\pi_{1} \pi^{k+1} \pi_{1}\right)^{m} \pi^{\prime} \rho_{\text {dev }}^{\prime}$. We define a strategy $f_{j}^{\prime}$ for $j$ such that $\rho\left(f_{-j}^{*}, f_{j}^{\prime}\right)=\pi_{0}\left(\pi_{1} \pi^{k} \pi_{1}\right)^{m} \pi^{\prime} \rho_{d e v}^{\prime}$. Now every history is of the form $\eta=$ $\pi_{c o m}^{\prime \prime} \pi_{d e v}^{\prime \prime}$, where $\pi_{c o m}^{\prime \prime}$ is the maximal common prefix of $\eta$ and $\pi_{0}\left(\pi_{1} \pi^{k+1} \pi_{1}\right)^{m} \pi^{\prime} \rho_{d e v}^{\prime}$. Given $\eta=$ $\pi_{c o m}^{\prime \prime} \pi_{d e v}^{\prime \prime}$, define

$$
\hat{\pi}_{c o m}^{\prime \prime}= \begin{cases}\pi_{c o m}^{\prime \prime} & \text { if } \pi_{c o m}^{\prime \prime}<\pi_{0}, \\ \pi_{0}\left(\pi_{1} \pi^{k+1} \pi_{2}\right)^{\ell} \pi^{\prime \prime} & \text { if } \pi_{c o m}^{\prime \prime}=\pi_{0}\left(\pi_{1} \pi^{k} \pi_{2}\right)^{\ell} \pi^{\prime \prime}, 0 \leq \ell \leq m, \\ & \text { and } \pi^{\prime \prime} \leq \pi_{1} \pi^{k} \pi_{2} .\end{cases}
$$

Subsequently, define $f_{j}^{\prime}$ such that for every history $\eta=\pi_{c o m}^{\prime \prime} \pi_{d e v}^{\prime \prime}$,

$$
f_{j}^{\prime}\left(\pi_{c o m}^{\prime \prime} \pi_{d e v}^{\prime \prime}\right)=g_{j}^{\prime}\left(\hat{\pi}_{c o m}^{\prime \prime} \pi_{d e v}^{\prime \prime}\right) \text {. }
$$


Defined thus, $\rho\left(f_{-j}^{*}, f_{j}^{\prime}\right)=\pi_{0}\left(\pi_{1} \pi^{k} \pi_{1}\right)^{m} \pi^{\prime} \rho_{d e v}^{\prime}$. As $n_{\gamma_{j}}<k$, it now follows that $\rho\left(f_{-j}^{*}, f_{j}^{\prime}\right)=\gamma_{j}$. This would again signify that $f^{*}$ is not a Nash equilibrium, a contradiction.

Finally, for the "if"-direction of $(i i)$, let $f^{*}=\left(f_{1}^{*}, \ldots, f_{n}^{*}\right)$ be a Nash equilibrium such that $\rho\left(f^{*}\right)=$ $\pi_{0}\left(\pi_{1} \pi^{k+1} \pi_{2}\right)^{\omega}$. We define another strategy profile $g^{*}=\left(g_{1}^{*}, \ldots, g_{n}^{*}\right)$ with $\rho\left(g^{*}\right)=\pi_{0}\left(\pi_{1} \pi^{k} \pi_{2}\right)^{\omega}$ and show that it is a Nash equilibrium as well. Note that every history has the form $\eta=\pi_{\text {com }} \pi_{d e v}$, where $\pi_{c o m}$ is the maximal common prefix of $\eta$ and $\pi_{0}\left(\pi_{1} \pi^{k} \pi_{2}\right)^{\omega}$, whereas $\pi_{d e v}$ is some 'deviant' continuation. Given $\eta=\pi_{c o m} \pi_{d e v}$, moreover, either $\pi_{\text {com }}<\pi_{0}$ or $\pi_{c o m}=\pi_{0}\left(\pi_{1} \pi^{k} \pi_{2}\right)^{\ell} \pi^{\prime}$ for some $0 \leq \ell \leq m$ and $\pi^{\prime} \leq \pi_{1} \pi^{k} \pi_{2}$. If the former, define $\hat{\pi}_{c o m}=\pi_{c o m}$. If the latter, that is, if $\pi_{c o m}=$ $\pi_{0}\left(\pi_{1} \pi^{k} \pi_{2}\right)^{\ell} \pi^{\prime}$, let

$$
\hat{\pi}_{\text {com }}= \begin{cases}\pi_{0}\left(\pi_{1} \pi^{k+1} \pi_{2}\right)^{\ell} \pi^{\prime} & \text { if } \pi^{\prime}<\pi_{1} \pi^{k}, \\ \pi_{0}\left(\pi_{1} \pi^{k+1} \pi_{2}\right)^{\ell} \pi_{1} \pi^{k+1} \pi^{\prime} & \text { if } \pi^{\prime} \leq \pi_{2}\end{cases}
$$

Subsequently, define for every player and every history $\eta=\pi_{c o m} \pi_{d e v}$,

$$
g_{i}^{*}\left(\pi_{c o m} \pi_{d e v}\right)=f_{i}^{*}\left(\hat{\pi}_{c o m} \pi_{d e v}\right) .
$$

Then, $\rho\left(g^{*}\right)=\pi_{0}\left(\pi_{1} \pi^{k} \pi_{2}\right)^{\omega}$.

Now assume for a contradiction that $g^{*}$ is not a Nash equilibrium. Then, there is some player $j$ with $\rho\left(g^{*}\right) \not \models \gamma_{j}$ and some strategy $g_{j}^{\prime}$ for $j$ such that $\rho\left(g_{-j}^{*}, g_{j}^{\prime}\right) \models \gamma$. As $n_{\neg \gamma_{j}}<k$, then also $\rho\left(f^{*}\right) \not \models$ $\gamma_{j}$. Let $\rho\left(g_{-j}^{*}, g_{j}^{\prime}\right)=\pi_{c o m}^{\prime} \rho_{d e v}^{\prime}$, where $\pi_{c o m}^{\prime}$ is the maximal common prefix of $\rho\left(g^{*}\right)$ and $\rho\left(g_{-j}^{*}, g_{j}^{\prime}\right)$, and $\rho_{d e v}^{\prime}$ some 'deviant' continuation.

If $\pi_{\text {com }}^{\prime}<\pi_{0}$, then $\rho\left(f_{-j}^{*}, g_{j}^{\prime}\right)=\rho\left(g_{-j}^{*}, g_{j}^{\prime}\right)$. Hence, $\rho\left(f_{-j}^{*}, g_{j}^{\prime}\right) \models \gamma_{j}$, and it would follow that $f^{*}$ is not a Nash equilibrium. If, on the other hand, $\pi_{c o m}^{\prime}=\pi_{0}\left(\pi_{1} \pi^{k} \pi_{2}\right)^{\ell} \pi^{\prime}$ for some $\ell \geq 0$ and some $\pi^{\prime} \leq \pi_{1} \pi^{k} \pi_{2}$, then we define a strategy $f_{j}^{\prime}$ for $j$ such that $\rho\left(f_{-j}^{*}, f_{j}^{\prime}\right)=\pi_{0}\left(\pi_{1} \pi^{k+1} \pi_{2}\right)^{\ell} \pi^{\prime} \rho_{d e v}^{\prime}$. To this end, note that every history is of the form $\eta=\pi_{c o m}^{\prime \prime} \pi_{d e v}^{\prime \prime}$, where $\pi_{c o m}^{\prime \prime}$ is the maximal common prefix of $\eta$ and $\pi_{0}\left(\pi_{1} \pi^{k+1} \pi_{2}\right)^{m} \pi^{\prime} \rho_{d e v}^{\prime}$, and $\pi_{d e v}^{\prime \prime}$ some 'deviant' continuation. Given $\eta=\pi_{c o m}^{\prime \prime} \pi_{d e v}^{\prime \prime}$, define $\check{\pi}_{c o m}^{\prime \prime}=\pi_{c o m}^{\prime \prime}$ if $\pi_{c o m}^{\prime \prime}<\pi_{0} \pi^{k}$. Otherwise, that is if $\pi_{c o m}^{\prime \prime}=\pi_{0}\left(\pi_{1} \pi^{k+1} \pi_{1}\right)^{m} \pi^{\prime \prime}$ for some $0 \leq \ell \leq m$ and $\pi^{\prime \prime} \leq \pi_{1} \pi^{k+1} \pi_{2}$, let

$$
\check{\pi}_{c o m}^{\prime \prime}= \begin{cases}\pi_{0}\left(\pi_{1} \pi^{k} \pi_{2}\right)^{\ell} \pi^{\prime \prime} & \text { if } \pi^{\prime \prime}<\pi_{1} \pi^{k} \\ \pi_{0}\left(\pi_{1} \pi^{k} \pi_{2}\right)^{\ell} \pi_{1} \pi^{k-1} \pi^{\prime \prime \prime} & \text { if } \pi^{\prime \prime}=\pi_{0} \pi^{k} \pi^{\prime \prime \prime} \text { and } \pi^{\prime \prime \prime} \leq \pi^{k} \pi_{2} .\end{cases}
$$

Now define for all histories $\eta=\pi_{c o m}^{\prime \prime} \pi_{d}^{\prime \prime} e v$,

$$
f_{j}^{\prime}\left(\pi_{c o m}^{\prime \prime} \pi_{d e v}^{\prime \prime}\right)=g_{j}^{\prime}\left(\check{\pi}_{c o m}^{\prime \prime} \pi_{d e v}^{\prime \prime}\right) .
$$

Then, $\rho\left(f_{-j}^{*}, f_{j}^{\prime}\right)=\pi_{0}\left(\pi_{1} \pi^{k+1} \pi_{2}\right)^{\ell} \rho_{d e v}^{\prime}$. As $n_{\gamma_{j}}<k$, it follows that $\rho\left(f_{-j}^{*}, f_{j}^{\prime}\right) \models \gamma_{j}$, which implies that $f^{*}$ is not a Nash equilibrium, a contradiction.

As an immediate consequence of Proposition 4.6, we thus find that LTL cannot express in equilibrium every $\omega$-regular property, that is, LTL ${ }^{N E} \nsucceq \mathscr{L}_{\omega \text {-reg. }}$. For instance, the property expressed by the $\omega$-regular expression $(\varnothing ; \varnothing)^{*} ;\{p\}^{\omega}$ cannot be obtained as the set of equilibrium runs of any LTL-game.

In view of the characterisation result by Kučera and Strejček, Proposition 4.6 gives us one half of the proof that $\mathrm{LTL} \geq \mathrm{LTL}^{N E}$. To prove the second half-namely, that that the set of equilibrium runs of every LTL-game is an $\omega$-regular set-we adapt a result by [Gutierrez et al. 2017b], which provides us with a construction of a parity automata that recognises all words corresponding to the equilibrium runs of a given LTL-game. We saw above how Lemma 3.5 characterised the equilibrium runs $\rho$ of an iterated Boolean game $G$ as those for which there is a bi-partition $\{W, L\}$ of the players. such that $W$ is the group of agents that have goal achieved, whereas $L$ consists of those players for whom $\rho$ is consistent with a punishment strategy against them. We can now leverage this result apply the automata-theoretic approach to linear temporal logic as proposed by [Vardi 1996] so as to obtain the following proposition. 
Proposition 4.7 (AfTer [GUTIERREZ Et Al. 2017B]). For every LTL-game G, the set $N E(G)$ of equilibrium runs is $\omega$-regular.

PROOF SKETCH. The proof invokes the concepts of deterministic and non-deterministic Rabin word- and tree automata. For the formal definitions of these, the reader is referred to [Perrin and Pin 2004; Kupferman 2018; Löding 2012; Strejček 2004].

Let $\{W, L\}$ be any bipartition of the set of players. For every player $i$ in $W$, we can construct a non-deterministic Rabin word automaton $A_{\gamma_{i}}$ that recognises all runs satisfying $\gamma_{i}$. In a slightly more complicated way we can also construct for each player $j \in L$ a non-deterministic Rabin word automaton $B_{\gamma_{j}}$ that recognises all runs that are consistent with a punishment strategy against $j$. First, consider the non-deterministic Rabin word automaton $\bar{A}_{\gamma_{j}}$ that recognises all runs not satisfying $\gamma_{j}$. By virtue of Theorem 2 in [Pnueli and Rosner 1989], we know there exists a non-deterministic Rabin automaton on trees $\bar{A}_{\gamma_{j}}^{\prime}$ recognising all trees that represent the execution of a winning strategy by the coalition $N \backslash\{j\}$ against $j$. Now observe that the branches of the trees recognised by $\bar{A}_{\gamma_{j}}^{\prime}$ correspond exactly to those runs of $G$ that are consistent with a punishment strategy against $j$. Moreover, as an immediate consequence of Corollary 17 in [Niwinski and Walukiewicz 1998], these branches are recognised by a non-deterministic Rabin word-automaton $B_{\gamma_{j}}$. As the languages recognised by non-deterministic Rabin word-automaton are closed under union and intersection, there exists a non-deterministic Rabin word automaton $A_{N E}$ that recognises exactly those runs $\rho$ of $G$ for which there is some bipartition $\{W, L\}$ of the players such that $\rho$ satisfies the goal of each player in $W$ and is consistent with a punishment strategy against each player in $L$. By Lemma 3.5 it follows that that $A_{N E}$ recognises the Nash equilibrium runs of $G$, and we may conclude that $N E(G)$ is $\omega$-regular.

Together with Theorem 4.5, Propositions 4.6 and 4.7 immediately yield that LTL can express every temporal property in formulas that LTL can express in equilibrium. That also the opposite direction holds is an immediate consequence of Proposition 4.1, provided that there are are least two propositional variables. Hence, we obtain the main result of this subsection.

THEOREM 4.8. Let $|\Phi| \geq 2$. Then, $\operatorname{LTL}(\Phi)$ is just as expressive in formulas as it is in equilibrium, that is, $\operatorname{LTL}(\Phi) \equiv \operatorname{LTL}^{N E}(\Phi)$.

Proof. Having assumed $|\Phi| \geq 2$, we obtain $\operatorname{LTL}^{N E}(\Phi) \geq \operatorname{LTL}(\Phi)$ as an immediate consequence of Proposition 4.1. To see that also LTL $\geq \mathrm{LTL}^{N E}$, consider an arbitrary temporal property $X$ that is expressed in equilibrium by LTL, that is, $X$ is expressed by LTL ${ }^{N E}$. Then, there is an iterated Boolean game $G$ such that $X$ is the set of Nash equilibrium runs of $G$. Propositions 4.6 and 4.7 yield that $X$ is non-counting and $\omega$-regular, respectively. By virtue of Theorem 4.5, we may then conclude that LTL can express $X$ in formulas as well.

\subsection{Stutter-invariant Specifications: The Next-free Fragment $\mathscr{L}_{U}$}

We conclude this section by investigating the expressive power in equilibrium of the next-free or maximally stutter-invariant fragment $\mathscr{L}_{\mathrm{U}}$. In the verification and model checking literature, this fragment plays a prominent role because it guarantees stutter-invariant specifications of programs and concurrent systems, without losing expressiveness otherwise. In this section, we find that there nevertheless are stutter-sensitive properties that $\mathscr{L}_{U}$ can express in equilibrium, even though it cannot express all properties that are expressed by LTL in formulas. That is, LTL $>\mathscr{L}_{\mathrm{U}}^{N E}>\mathscr{L}_{\mathrm{U}}$ (provided that there are at least two propositional variables).

Recall that formally a temporal property $X \subseteq$ runs $_{\Phi}$ is said to be stutter-invariant if, for all runs $\rho=v_{0} v_{1} v_{2}, \ldots$ and every sequence $k_{0}, k_{1}, k_{2}, \ldots$ of positive integers,

$$
v_{0} v_{1} v_{2} \ldots \in X \text { if and only if } v_{0}^{k_{0}} v_{1}^{k_{1}} v_{2}^{k_{2}} \ldots \in X \text {, }
$$

where $v^{k}$ denotes the $k$-fold iteration of $v$. Thus, for instance, consider the property defined by the $\omega$-regular expression $(p ; \varnothing)^{\omega}$, henceforth denoted by toggle $(p)$. This property does not define a 
stutter-free property, as it contains the run $\{p\} \varnothing\{p\} \varnothing \ldots$, but not the run $\{p\}\{p\} \varnothing \varnothing\{p\}\{p\} \varnothing \varnothing \ldots$ Note that toggle $(p)$ is expressed by the LTL-formula $p \wedge \mathrm{G}(p \leftrightarrow \mathrm{X} \neg p)$; it cannot be expressed by a formula of $\mathscr{L}_{U}$ though. In a seminal paper, [Peled and Wilke 1997] showed that the so-called next-free fragment $\mathscr{L}_{U}$ is the largest stutter-free fragment of LTL, that is, $\mathscr{L}_{U}$ can express every stutter-free property that LTL can express in formulas, even when also using the next-operator $\mathrm{X}$ [Etessami 2000].

[Lamport 1983] makes a case in favour of stutter-invariant specifications and, in particular, vehemently argued against the inclusion of the next-operator $X$ in the syntax of temporal logic. The main reason for his opposition is the observation that temporal logics are meant to facilitate reasoning about abstract specifications of programs rather than about their concrete implementations, and that the next-operator enables reasoning about irrelevant aspects of the implementation of a program specification. Lamport gives the example of requiring that a program take exactly 17 steps to implement a queue, which relates to the specification, but can only be expressed by stutter-sensitive fragments. He furthermore alleged that stutter-invariant specifications still enable reasoning about "the next state in which a significant change occurs [...] at the level of detail of the specification" [Lamport 1983, page 661]. Accordingly, including the next-operator would run counter to modular or hierarchical approaches towards program specification: "you will see that increasing the expressiveness of our temporal logic with a next operator would destroy the entire logical foundation for its use in hierarchical methods" (ibid., page 661).

Elaborating on the topic of stutter-invariant specifications, [Peled and Wilke 1997] furthermore point to the advantages of stutter-invariant specification of concurrent programs, where the different ways in which processes can be interleaved is seen as a being essentially part of the implementation. This invariance under arbitrary interleavings is also the technical basis for state space reduction techniques for model checking concurrent system.

We find that the stutter-invariance of properties expressed by $\mathscr{L}_{U}$ does not extend to the temporal properties that can be expressed by $\mathscr{L}_{U}$ in equilibrium. To see this, let $G_{\text {toggle }(p)}$ be the iterated Boolean game on a set $\Phi$ of propositional variables containing $p$ and $q$, and two players, $i$ and $j$, such that:

$$
\begin{aligned}
\Phi_{i} & =\{p\} \\
\Phi_{j} & =\Phi \backslash\{p\} \\
\gamma_{i} & =p \wedge \neg \gamma_{j} \\
\gamma_{j} & =\mathrm{F}((p q \wedge p q \cup p \bar{q}) \vee(\bar{p} q \wedge \bar{p} q \cup \bar{p} \bar{q}) \vee(p \bar{q} \wedge p \bar{q} \bigcup p q) \vee(\bar{p} \bar{q} \wedge \bar{p} \bar{q} \bigcup \bar{p} q))
\end{aligned}
$$

Note that, for $\gamma_{j}$ to be satisfied, variable $p$ has to assume the same truth-value at at least two consecutive times. The only way player $i$ can prevent this from happening, and thus hope to satisfy her goal, is by single-handedly toggling the truth-value of $p$ starting by setting $p$ to true. On this basis we can show that the equilibria runs of $G_{t o g g l e}(p)$ are given exactly by toggle $(p)$. Hence, $\mathscr{L}_{U}$ can express stutter-sensitive properties in equilibrium.

This is interesting, because, intuitively, the Nash equilibria of a multi-agent system of which the players are assumed to interact strategically in pursuit of their $\mathscr{L}_{U}$-goals does not seem to pertain to the specifics of how the constituent agents are implemented. As such, the result below may cast a new light on the desirability of stutter-invariant specifications of multi-agent systems. Later, we will see, however, that the expressiveness of $\mathscr{L}_{U}$ in equilibrium does not quite equal the expressive power of $\mathscr{L}_{U}$ in formulas with the next-operator $\mathrm{X}$ added, that is, the expressive power of LTL in formulas.

Proposition 4.9. Let $p, q \in \Phi$. Then, $N E\left(G_{\text {toggle }}(p)\right)=$ toggle $(p)$. Hence, $\mathscr{L}_{\mathrm{U}}(\Phi)$ expresses $\operatorname{tog} g$ le $(p)$ in equilibrium.

PROOF. It suffices to show that $N E\left(G_{\text {toggle }(p)}\right)=\operatorname{toggle}(p)$. To this end, consider an arbitrary run $\rho \in \operatorname{runs}_{\Phi}$ that toggles $p$, that is, such that $\rho, t \models p$ if and only if $t$ is even. We first observe that $\rho \not \models \gamma_{j}$. To see this, consider an arbitrary $t \geq 0$ and assume $\rho, t \models p q$. Then, $\rho, t+1 \not \models p$. Now 
consider an arbitrary $t^{\prime} \geq t$ with $\rho, t^{\prime} \models p \bar{q}$. Hence, note that $t^{\prime} \geq t+2$, and, as $\rho, t+1 \not \models p q$, we obtain $\rho, t \not \models p q \wedge p q \cup p \bar{q}$. Assuming instead that $\rho, t \models \bar{p} q, \rho, t \models p \bar{q}$, or $\rho, t \models \bar{p} \bar{q}$ an analogous arguments yield $\rho, t \not \models \bar{p} q \wedge \bar{p} q \cup \bar{p} \bar{q}, \rho, t \not \models p \bar{q} \wedge p \bar{q} \cup p q$, and $\rho, t \not \models \bar{p} \bar{q} \wedge \bar{p} \bar{q} \cup \bar{p} q$, respectively. We may conclude that $\rho \not \models \rho \forall \gamma_{j}$.

Now, consider an arbitrary run $\rho=v_{0} v_{1} v_{2} v_{3} \ldots$ in $\operatorname{runs}_{\Phi}$ that toggles $p$. We prove that $\rho$ is sustained by an equilibrium, that is, $\rho \in N E\left(G_{\operatorname{toggle}(p)}\right)$. To this end, define strategy $f_{i}^{\text {toggle }(p)}$ for player $i$ such that $f_{i}^{\text {toggle }(p)}(\varepsilon)=\{p\}$ and for paths $v_{0} \ldots v_{t}$ with $t \geq 0$,

$$
f_{i}^{\text {toggle }(p)}\left(v_{0} \ldots v_{t}\right)= \begin{cases}\{p\} & \text { if } t \text { is odd } \\ \varnothing & \text { otherwise }\end{cases}
$$

For player $j$ define strategy $f_{j}$ such that $f_{j}(\varepsilon)=v_{0} \cap \Phi_{j}$ and $f_{j}\left(v_{0}, \ldots, v_{k}\right)=v_{k+1} \cap \Phi_{j}$, if $k \geq 0$. Defined thus, it can easily be seen that $\rho\left(f_{i}^{\text {toggle }(p)}, f_{j}\right)=v_{0} v_{1} v_{2} \ldots$ Also, observe that by definition of $f_{i}$, we find that $\rho\left(f_{i}^{\text {toggle }(p)}, g_{j}\right)$ toggles $p$ for every strategy $g_{j}$ for player $j$. Hence $\rho\left(f_{i}^{\text {toggle }(p)}, g_{j}\right) \not \gamma_{j}$ for every strategy $g_{j}$ for player $j$ as well. It follows that player $j$ does not want to deviate from $\left(f_{i}^{\text {toggle }(p)}, f_{j}\right)$. Moreover, $\rho\left(f_{i}^{\text {toggle }(p)}, f_{j}\right) \models p$ as well as $\rho\left(f_{i}^{\text {toggle }(p)}, f_{j}\right) \not \gamma_{j}$. Hence, $\rho\left(f_{i}^{\text {toggle }(p)}, f_{j}\right)=\gamma_{i}$, and player $i$ does not want to deviate from $\left(f_{i}^{\text {toggle }(p)}, f_{j}\right)$ either. It follows that $\left(f_{i}^{\text {toggle }(p)}, f_{j}\right)$ is a Nash equilibrium sustaining $\rho$.

To conclude the proof, consider an arbitrary run $\rho^{\prime}=v_{0}^{\prime} v_{1}^{\prime} v_{2}^{\prime} \ldots$ that does not toggle $p$ and and equally arbitrary strategy profile $g=\left(g_{i}, g_{j}\right)$ with $\rho\left(g_{i}, g_{j}\right)=\rho^{\prime}$. Then there is a $t \geq 0$ such that either $(i)$ both $\rho, t=p$ and $\rho^{\prime}, t+1 \models p$ or $(i i)$ both $\rho^{\prime}, t \models \bar{p}$ and $\rho^{\prime}, t+1 \models \bar{p}$.

If $(i)$, and $\rho^{\prime} \not \models \gamma_{i}$, then $\rho\left(f_{i}^{\text {toggle }(p)}, g_{j}\right) \models \gamma_{i}$, that is, player $i$ wants to deviate from $g=\left(g_{i}, g_{j}\right)$ and get her goal achieved. On the other hand, if $\rho^{\prime} \models \gamma_{i}$, then $\rho^{\prime} \not \models \gamma_{j}$. In that case, we may assume without loss of generality that $\rho^{\prime}, t \models p q$, and define strategy $g_{j}^{\prime}$ for player $j$ such that for all $g_{j}^{\prime}(\varepsilon)=$ $g_{j}(\varepsilon)$ and, for paths $w_{0} \ldots w_{k}$ with $k \geq 0$,

$$
g_{j}^{\prime}\left(w_{0} \ldots w_{k}\right)= \begin{cases}g_{j}\left(w_{0} \ldots w_{k}\right) & \text { if } k<t, \\ \varnothing & \text { otherwise }\end{cases}
$$

Let $\rho\left(g_{i}, g_{j}^{\prime}\right)=v_{0}^{\prime \prime} v_{1}^{\prime \prime} v_{2}^{\prime \prime} \ldots$ Now observe that, $v_{0}^{\prime \prime} \ldots v_{t}^{\prime \prime}=v_{0}^{\prime} \ldots v_{t}^{\prime}$. Hence, $\rho\left(g_{i}, g_{j}^{\prime}\right), t \models p q$ Observe moreover that both $p \in g_{i}\left(v_{0}^{\prime \prime} \ldots v_{t}^{\prime \prime}\right)$ and $q \notin g_{j}^{\prime}\left(v_{0}^{\prime \prime} \ldots v_{t}^{\prime \prime}\right)$. Therefore also $\rho\left(g_{i}, g_{j}^{\prime}\right), t+1=p \bar{q}$. Accordingly, $\rho\left(g_{i}, g_{j}^{\prime}\right), t \models p q \wedge p q \cup p \bar{q}$, and hence $\rho\left(g_{i}, g_{j}^{\prime}\right) \models \gamma_{j}$. It follows that $g=\left(g_{i}, g_{j}\right)$ is not a Nash equilibrium. If $(i i)$, the argumentation is analogous, and, as $g=\left(g_{i}, g_{j}\right)$ had been chosen arbitrarily, we may conclude that $\rho^{\prime} \notin N E\left(G^{\text {toggle }(p)}\right)$, as desired.

As an almost immediate consequence of Proposition 4.9, we obtain that the stutter-invariant fragment $\mathscr{L}_{U}$ is strictly more expressive in equilibrium that it is in formulas.

THEOREM 4.10. Let $|\Phi| \geq 2$. Then $\mathscr{L}_{\mathrm{U}}$ is strictly more expressive in equilibrium than it in formulas, that is, $\mathscr{L}_{\mathrm{U}}^{N E}(\Phi)>\mathscr{L}_{\mathrm{U}}(\Phi)$.

ProOF. As we may assume that $|\Phi| \geq 2$, by Proposition 4 . 1 , we immediately obtain $\mathscr{L}_{\mathrm{U}}^{N E} \geq \mathscr{L}_{\mathrm{U}}$. For $p$ a propositional variable in $\Phi$, moreover, the stutter-sensitive property toggle $(p)$ cannot be expressed by $\mathscr{L}_{U}$ as this fragment is the largest stutter-invariant fragment of LTL. By Proposition 4.9, however, toggle $(p)$ can be expressed in equilibrium by $\mathscr{L}_{\mathrm{U}}$. Hence, $\mathscr{L}_{\mathrm{U}}^{N E}>\mathscr{L}_{\mathrm{U}}$.

Even though $\mathscr{L}_{U}$ can express in equilibrium some stutter-sensitive LTL-properties, its expressive power in equilibrium does not match that of full LTL in formulas. We thus find, quite strikingly, that $\mathscr{L}_{\mathrm{U}}$ cannot even express in equilibrium the property characterised by the LTL-formula X $p$. 
PROPOSITION 4.11. Let $p$ be a propositional variable contained in $\Phi$. Then, the fragment $\mathscr{L}_{U}(\Phi)$ cannot express in equilibrium the temporal property characterised by the LTLformula $\mathrm{X} p$.

PROOF. Recall that a run $\rho=v_{0} v_{1} v_{2} \ldots$ satisfies $\mathrm{X} p$ if and only if $p \in v_{1}$. Assume for a contradiction that $G$ is an $\mathscr{L}_{\mathrm{U}}$-game such that $N E(G)=\operatorname{runs}_{\Phi}(\mathrm{X} p)$. Consider a run $\rho=v_{0} v_{0} v_{1} v_{2} \ldots$ where $p \in v_{0}$ and $p \notin v_{1}$. As $\rho \in \operatorname{runs}_{\Phi}(\mathrm{X} p)$, there is an equilibrium $f^{*}=\left(f_{1}^{*}, \ldots, f_{n}^{*}\right)$ that sustains $\rho$, that is $\rho=\rho\left(f^{*}\right)$. Observe that $f_{i}^{*}(\varepsilon)=f_{i}^{*}\left(v_{0}\right)$ for all players $i$. Now define the strategy profile $f=\left(f_{1}, \ldots, f_{n}\right)$ such that for every player $i$ we have $f_{i}(\varepsilon)=f_{i}^{*}\left(v_{0}\right)$ and, for every $x_{0}, \ldots, x_{k}$ with $k \geq 0$,

$$
f_{i}\left(x_{0}, \ldots, x_{k}\right)= \begin{cases}f_{i}^{*}\left(v_{0}, x_{0}, \ldots, x_{k}\right) & \text { if } x_{0}=v_{0} \\ f^{*}\left(x_{0}, \ldots, x_{k}\right) & \text { otherwise. }\end{cases}
$$

A straightforward induction establishes that $\rho(f)=v_{0} v_{1} v_{2} v_{3} \ldots$. Having assumed that $p \notin v_{1}$, clearly $\rho(f) \notin \operatorname{runs}_{\Phi}(\mathrm{X} p)$. Hence, $f=\left(f_{1}, \ldots, f_{n}\right)$ is not an equilibrium of $G$ and there is some player $j$ such that $\rho(f) \not \forall \gamma_{j}$ and some strategy $g_{j}$ for $j$ such that $\rho\left(f_{-j}, g_{j}\right) \models \gamma_{j}$. As $\gamma_{j}$ expresses a stutter-invariant property, observe that now also $\rho\left(f^{*}\right) \not \neq \gamma_{j}$. Let $\rho\left(f_{-j}, g_{j}\right)=u_{0} u_{1} u_{2} \ldots$.

At this point, define strategy $g_{j}^{\prime}$ such that $g_{j}^{\prime}(\varepsilon)=g_{j}(\varepsilon)$ and, for every path $x_{0} \ldots x_{k}$,

$$
g_{j}^{\prime}\left(x_{0} \ldots x_{k}\right)= \begin{cases}g_{j}\left(x_{1} \ldots x_{k}\right) & \text { if } x_{0}=v_{0}, \\ g_{j}\left(x_{0} \ldots x_{k}\right) & \text { otherwise, }\end{cases}
$$

on the understanding that $x_{1} \ldots x_{0}=\varepsilon$. Let $\rho\left(f_{-j}^{*}, g_{j}^{\prime}\right)=w_{0} w_{1} w_{2} w_{3} \ldots$

At this point, we distinguish two cases. In either one we show that $\rho\left(f_{-j}^{*}, g_{j}^{\prime}\right) \models \gamma_{j}$. Having already seen that $\rho\left(f^{*}\right) \not \forall \gamma_{j}$, this then contradicts that $f^{*}$ is a Nash equilibrium.

First assume that $g_{j}(\varepsilon)=f_{j}(\varepsilon)$, that is, player $j$ does not deviate from $f_{j}$ immediately. Hence, $u_{0}=v_{0}$. We show that $\rho\left(f_{-j}^{*}, g_{j}^{\prime}\right)=u_{0} u_{0} u_{1} u_{2} \ldots$, that is, $w_{0}=u_{0}$ and $w_{t}=u_{t-1}$ for all $t \geq 1$. Since, $\rho\left(f_{-j}, g_{j}\right) \models \gamma_{j}$ and $\gamma_{j}$ is a stutter-invariant property, it follows that also $\rho\left(f_{-j}^{*}, g_{j}^{\prime}\right) \models \gamma_{j}$, a contradiction.

Observe that both $g_{j}^{\prime}(\varepsilon)=g_{j}(\varepsilon)=f_{j}(\varepsilon)$ and $f_{i}^{*}(\varepsilon)=f_{i}^{*}\left(v_{0}\right)=f_{i}(\varepsilon)$ for all $i \neq j$. Hence, $w_{0}=v_{0}$, and therefore $w_{0}=u_{0}$.

We now show by induction that $w_{t}=u_{t-1}$ for all $t \geq 1$. If $t=1$, observe that $g_{j}^{\prime}\left(w_{0}\right)=g_{j}(\varepsilon)$, as $w_{0}=v_{0}$. Moreover, $f_{i}^{*}\left(w_{0}\right)=f_{i}^{*}\left(v_{0}\right)=f_{i}(\varepsilon)$ for all $i \neq j$. It follows that $w_{1}=u_{0}$, as desired. For the induction step, we may assume that $w_{0} \ldots w_{t}=u_{0} u_{0} \ldots u_{t-1}$. Then, $g_{j}^{\prime}\left(w_{0} \ldots w_{t}\right)=g_{j}\left(w_{1} \ldots w_{t}\right)$ as $w_{0}=v_{0}$, and hence, by the induction hypothesis, $g_{j}^{\prime}\left(w_{0} \ldots w_{t}\right)=g_{j}\left(u_{0} \ldots u_{t-1}\right)$. Moreover, for all players $i \neq j$, we have $f_{i}^{*}\left(w_{0} \ldots w_{t}\right)=f_{i}\left(w_{1} \ldots w_{t}\right)$ because $w_{0}=v_{0}$, and hence, by the induction hypothesis, $f_{i}^{*}\left(w_{0} \ldots w_{t}\right)=f_{i}\left(u_{0} \ldots u_{t-1}\right)$. It follows that $w_{t+1}=u_{t}$, as desired.

Finally, assume that $g_{j}(\varepsilon) \neq f_{j}(\varepsilon)$, and hence $u_{0} \neq v_{0}$. We prove by induction that $w_{t}=u_{t}$ for all $t \geq 0$, that is, $\rho\left(f_{-j}^{*}, g_{j}^{\prime}\right)=\rho\left(f_{-j}, g_{j}\right)$. It then immediately follows that $\rho\left(f_{-j}^{*}, g_{j}^{\prime}\right) \models \gamma_{j}$. For the basis with $t=0$, observe that both $g_{j}^{\prime}(\varepsilon)=g_{j}(\varepsilon)$ and $f_{i}^{*}(\varepsilon)=f_{i}^{*}\left(v_{0}\right)=f_{i}(\varepsilon)$. It follows that $w_{0}=u_{0}$. For the induction step we ma assume that $w_{0} \ldots w_{t}=u_{0} \ldots u_{t}$. Recall that $u_{0} \neq v_{0}$. Hence, $g_{j}^{\prime}\left(w_{0} \ldots w_{t}\right)=g^{\prime}\left(u_{0} \ldots u_{t}\right)=g_{j}\left(u_{0} \ldots u_{t}\right)$, as well as $f_{i}^{*}\left(w_{0} \ldots w_{t}\right)=f_{i}^{*}\left(u_{0} \ldots u_{t}\right)=f_{i}\left(u_{0} \ldots u_{t}\right)$ for all $i \neq j$. We may conclude that $w_{t+1}=u_{t+1}$, as desired.

We are now in a position to conclude this section by showing that LTL is still strictly more expressive in formulas than $\mathscr{L}_{U}$ in equilibrium.

THEOREM 4.12. Let $|\Phi| \geq 2$. Then, LTL is strictly more expressive in formulas than $\mathscr{L}_{\mathrm{U}}$ in equilibrium, that is, $\operatorname{LTL}(\Phi)>\mathscr{L}_{\mathrm{U}}^{N E}(\Phi)$.

ProOF. As LTL $\geq \mathscr{L}_{\mathrm{U}}$, by Proposition 4.3 also $\mathrm{LTL}^{N E} \geq \mathscr{L}_{\mathrm{U}}^{N E}$. Having assumed that $|\Phi| \geq$ 2, Theorem 4.8 then entails that LTL $\geq \mathscr{L}_{\mathrm{U}}^{N E}$. Proposition 4.11, moreover, shows that there are 
properties expressible by LTL in formulas that cannot be expressed by $\mathscr{L}_{U}$ in equilibrium. Hence, $\mathrm{LTL}>\mathscr{L}_{\mathrm{U}}^{N E}$.

\section{PROJECTIVE EXPRESSIVENESS}

In this section, we consider projective expressiveness in formulas along with the attendant concept of projective expressiveness in equilibrium. Projective expressiveness is weaker than regular expressiveness in that a property being expressible (either in formulas or in in equilibrium) implies that property to be projectively expressible, but not necessarily the other way round. We find that projective expressiveness in equilibrium is a very powerful concept, and as the main result of this section we show that the next-free fragment $\mathscr{L}_{U}$ can express in equilibrium every $\omega$-regular property.

\subsection{Projective Expressiveness in Formulas}

Projective expressiveness abstracts away from the propositional variables available in the language. Rather than requiring that a temporal property $X$ coincide with the set of runs satisfying some formula $\varphi$ in a fragment $\mathscr{L}(\Phi)$, it demands that $X$ be the set of projections to $\Phi$ of the runs satisfying some formula $\varphi$ of $\mathscr{L}$ in an extended set of propositional variables. Formally, we say that LTLfragment $\mathscr{L}(\Phi)$ can projectively express (in formulas) property $X \subseteq$ runs $_{\Phi}$ if there is some finite set $\Psi$ of auxiliary variables and some formula $\varphi \in \mathscr{L}(\Phi \cup \Psi)$ such that $X=\left\{\left.\rho\right|_{\Phi} \in \operatorname{runs}_{\Phi}: \rho \in\right.$ $\left.\operatorname{runs}_{\Phi \cup \Psi}(\varphi)\right\}^{2}$

The roots of projective expressiveness go back to the work of Beth [Beth 1953] and Craig [Craig $1957]$ in the 1950 s on definability in first order logic. The concept has numerous applications in model theory [Chang and Keisler 1990; Hodges 1993] and has recently also been studied in the context of modal and temporal logics [Gheerbrant and ten Cate 2009; Halpern et al. 2009]. In our setting, projective expressiveness defines relatively weak constraints on a fragment and should be carefully distinguished from standard expressiveness. Thus, recall that the properties that LTL can express in formulas are non-counting and cannot, for instance, characterise temporal property $\operatorname{even}(p)$, the set of runs on $2^{\Phi}$ in which $p$ is set to true at every even state [Wolper 1983]. Still, $q \wedge \mathrm{G}(q \leftrightarrow \mathrm{X} \neg q) \wedge \mathrm{G}(q \rightarrow p)$ projectively expresses exactly this property. It is known from the literature that every $\omega$-regular property can be projectively expressed by LTL, if one can use an unbounded number of additional propositional variables [Gheerbrant and ten Cate 2009; Thomas 1997]. We reformulate this result for our setting.

PROPOSITION 5.1. LTL can projectively express all $\omega$-regular properties.

Proof SKetCH. Let $A=\left(Q, 2^{\Phi}, \delta, Q_{0}, F\right)$ be a nondeterministic Büchi automaton [Baier and Katoen 2008]. We construct a formula $\varphi_{A}$ in $\operatorname{LTL}(\Phi \cup Q)$, where $\Phi$ and $Q$ are disjoint, as follows:

$$
\begin{aligned}
& \varphi_{\text {init }}^{A}=\bigvee_{q \in Q_{0}} q \\
& \varphi_{\text {trans }}^{A}=\mathrm{G}\left(\bigwedge_{q \in Q}\left(q \rightarrow \bigvee_{\left\{\left(q^{\prime}, v\right): q^{\prime} \in \delta(q, v)\right\}}\left(\chi_{v}^{\Phi} \wedge \mathrm{X} q^{\prime}\right)\right)\right) \\
& \varphi_{\text {accept }}^{A}=\mathrm{GF \bigvee} \bigvee_{q \in F} q \\
& \varphi_{\text {invar }}^{A}=\mathrm{G}\left(\bigvee_{q \in Q}\left(q \wedge \bigwedge_{q^{\prime} \neq q} \bar{q}^{\prime}\right)\right)
\end{aligned}
$$

Then set $\varphi^{A}=\varphi_{\text {init }}^{A} \wedge \varphi_{\text {trans }}^{A} \wedge \varphi_{\text {accept }}^{A} \wedge \varphi_{\text {invar }}^{A}$. By an inductive argument it then follows that $L_{A}=$ $\left\{\left.\rho\right|_{\Phi}: \rho \in \operatorname{runs}_{\Phi \cup Q}\left(\varphi_{A}\right)\right\}=\left.\operatorname{runs}_{\Phi}\left(\varphi_{A}\right)\right|_{\Phi}$, where $L_{A}$ is the language accepted by $A$. Recalling that the class of $\omega$-regular languages over $2^{\Phi}$ is exactly the class of languages accepted by some non-

\footnotetext{
${ }^{2}$ Our concept of projective expressiveness should be distinguished from that of projective properties as defined in [Peled 1997]. There, a property over $\left(\Sigma_{1} \times \cdots \times \Sigma_{n}\right)^{\omega}$ is said to be projective whenever two runs are in the property if and only if their stutter-free projections on each $\Sigma_{i}^{\omega}$ are identical. That means, that each of the $n$ components behave in a stutter-invariant fashion. Peled writes: "Thus for the temporal logics ETL and LTL properties using the correspondence between properties expressed in these logics and $\omega$-regular and star-free $\omega$-regular, respectively. Thus for the temporal logics LTL and ETL, the projective properties are always expressible as a boolean combination of local (stuttering-closed) properties."
} 
deterministic Büchi automaton, it follows that LTL can express projectively in formulas every $\omega$ regular property.

It is interesting to note that allowing for additional variables along with projection has a similar effect as, for instance, extending LTL to Extended Temporal Logic (ETL) by including in the syntax suitable grammar-operators as proposed in [Wolper 1983].

Moreover, all properties that LTL can express projectively are $\omega$-regular. To see this first observe that, if a temporal property $X \subseteq r u n s_{\Phi}$ is $\omega$-regular, then so is $\left.X\right|_{\Psi}$ for every $\Psi \subseteq \Phi$.

LEMMA 5.2. Let $X \subseteq$ runs $_{\Phi}$ be an $\omega$-regular property, and $\Psi \subseteq \Phi$. Then, $\left.X\right|_{\Psi}$ is also $\omega$-regular.

Proof. Let $A=\left(Q, 2^{\Phi}, \delta, Q_{0}, F\right)$ be a nondeterministic Büchi automaton accepting $X$. Define $\left.A\right|_{\Psi}=\left(Q, 2^{\Phi},\left.\delta\right|_{\Psi}, Q_{0}, F\right)$ as the non-deterministic Büchi automaton with $\left.\delta\right|_{\Psi}$ such that, for all $q, q^{\prime} \in Q$ and $v \in 2^{\Psi}$,

$$
\left.q^{\prime} \in \delta\right|_{\Psi}(q, v) \quad \text { if and only if } \quad q^{\prime} \in \delta\left(q, v \cup v^{\prime}\right) \text { for some } v^{\prime} \in 2^{\Phi \backslash \Psi} .
$$

It is then easy to see that $\left.A\right|_{\Phi}$ accepts $\left.X\right|_{\Phi}$. First consider an arbitrary $\omega$-word $\rho=v_{0} v_{1} v_{2} \ldots$ accepted by $\left.A\right|_{\Phi}$. Then consider an accepting sequence of states $q_{0} q_{1} q_{2} \ldots$ of $\left.A\right|_{\Phi}$ while reading $\rho$, that is, $\left.q_{t+1} \in \delta\right|_{\Phi}\left(q_{t}, v_{t}\right)$ for all $t \geq 0$. Then, $q_{0} q_{1} q_{2} \ldots$ is also an accepting sequence of states of $A$ while reading an $\omega$-word $\rho^{\prime}=v_{0}^{\prime} v_{1}^{\prime} v_{2}^{\prime} \ldots$ with $v_{t}=v_{t}^{\prime} \cap \Phi$ for every $t \geq 0$, that is, with $\left.\rho^{\prime}\right|_{\Phi}=\rho$. Hence, $\left.\rho \in X\right|_{\Phi}$.

Finally, consider an arbitrary $\omega$-word $\rho=v_{0} v_{1} v_{2} \ldots$ in $\left.X\right|_{\Phi}$. Then, there is an $\omega$-word $\rho^{\prime}=$ $v_{0}^{\prime} v_{1}^{\prime} v_{2}^{\prime} \ldots$ accepted by $A$ such that $\left.\rho^{\prime}\right|_{\Phi}=\rho$. Let $q_{0} q_{1} q_{2} \ldots$ be an accepting sequence of states visited by $A$ while reading $\rho^{\prime}$. In particular, $q_{t+1} \in \delta\left(q_{t}, v_{t}^{\prime}\right)$ for all $t \geq 0$. Then, however, also $q_{t+1} \in$ $\left.\delta\right|_{\Phi}\left(q_{t}, v_{t}^{\prime} \cap \Phi\right)$. Observing that $v_{t}^{\prime} \cap \Phi=v_{t}$ for all $t \geq 0$, we find that $q_{0} q_{1} q_{2} \ldots$ is also an accepting sequence of states visited by $\left.A\right|_{\Phi}$ while reading $\rho$. Hence, $\rho$ is accepted by $\left.A\right|_{\Phi}$. We may conclude that $\left.X\right|_{\Phi}$ is $\omega$-regular.

The following proposition now follows almost immediately.

PROPOSITION 5.3. All temporal properties that are projectively expressed by LTL in formulas are $\omega$-regular.

PROOF. Consider an arbitrary temporal property $X \subseteq r u n s_{\Phi}$ that LTL expresses projectively. Then, there is some auxiliary set of variables $\Psi$ and a formula $\varphi \in \operatorname{LTL}(\Phi \cup \Psi)$ such that $X=$ $\left.\operatorname{runs}_{\Phi \cup \Psi}(\varphi)\right|_{\Phi}$. Observe that $\operatorname{runs}_{\Phi \cup \Psi}(\varphi)$ is an $\omega$-regular property, and, hence, by Lemma 5.2, so is $X$.

\subsection{Projective Expressiveness in Equilibrium}

Projective expressiveness in equilibrium can now be defined analogously to how expressiveness in equilibrium was defined in Section 4.1 with respect to expressiveness in formulas. In a similar way as do the formulas of a temporal logic, the Nash equilibria of an iterated Boolean game also defines a linear time property as the set of runs they sustain. Given a set $\Phi$ of propositional variables, we thus say that LTL-fragment $\mathscr{L}(\Phi)$ projectively expresses in equilibrium temporal property $X \subseteq$ runs $_{\Phi}$, if there is some set of $\Psi$ of auxiliary propositional variables and an $\mathscr{L}(\Phi \cup \Phi)$-game $G$ such that $X=\left.N E(G)\right|_{\Phi}$. Recall that an $\mathscr{L}(\Phi \cup \Psi)$-game is an iterated Boolean game whose players' goals are given by formulas of $\mathscr{L}(\Phi \cup \Psi)$, and that $\left.N E(G)\right|_{\Phi}=\left\{\left.\rho\right|_{\Phi}: \rho \in N E(G)\right\}$. Note that, as one can always set $\Psi=\varnothing$, a property being expressible in equilibrium implies that that property can also be expressed projectively in equilibrium.

In the previous section, Proposition 4.1 esatablished that expressiveness in formulas implies expressiveness in equilibrium, provided that there are at least two propositional variables. For projective expressiveness this still holds, but without the caveat of $\Phi$ not being a singleton. 
PROPOSITION 5.4. Let $\mathscr{L}$ be an LTL-fragment on $\Phi$ and $X \subseteq$ runs $\Phi$ a temporal property over $2^{\Phi}$. Then, if $\mathscr{L}$ can projectively express $X$ in formulas, then $\mathscr{L}$ can also projectively express $X$ in equilibrium.

Proof. Assume that $\mathscr{L}(\Phi)$ projectively expresses $X \subseteq r u n s_{\Phi}$ in formulas. Then, there is an auxiliary set $\Psi$ of propositional variables and a formula $\varphi \in \mathscr{L}(\Phi \cup \Psi)$ such that $X=\left.\operatorname{runs}_{\Phi \cup \Psi}(\varphi)\right|_{\Phi}$. Let $p$ and $q$ be two variables disjoint from $\Phi \cup \Psi$, and let $X^{\prime}$ be defined as the set of all runs $\rho \in$ runs $_{\Phi \cup \Psi \cup\{p, q\}}$ such that $\rho \models \varphi$. Hence, $X^{\prime}$ is expressed by $\mathscr{L}(\Phi \cup \Psi \cup\{p, q\})$ in formulas. By Proposition 4.1, we find that $X^{\prime}$ can be expressed in equilibrium by $\mathscr{L}(\Phi \cup \Psi \cup\{p, q\})$. Observing that $X=\left.X\right|_{\Phi}$ finally yields that $X$ can be projectively expressed by $\mathscr{L}(\Phi)$ in equilibrium.

Using a construction similar to the matching-pennies game $G_{\varphi}^{m p}$, we can furthermore show that, if a fragment $\mathscr{L}$ can express a property $X$ in formulas, it can also projectively express the complement of $X$ in equilibrium. This result is especially relevant for fragments that are not closed under negation.

PROPOSITION 5.5. Let $X \subseteq$ runs $_{\Phi}$ be a temporal property that can be expressed in formulas by fragment $\mathscr{L}$. Then, $\mathscr{L}$ can projectively express runs $s_{\Phi} \backslash X$ in equilibrium.

ProOF. Let $p, q \notin \Phi$ and let $X$ be expressed by the $\mathscr{L}$-formula $\varphi$. Consider the two-player $\mathscr{L}$ game $G$ with $\Phi_{i}=\{p\}, \Phi_{j}=\Phi \cup\{q\}$ and the players' goals being given by $\gamma_{i}=\varphi \wedge(p \leftrightarrow q)$ and $\gamma_{j}=\bar{p} \leftrightarrow q$. First observe that no equilibrium run in $G$ satisfies $\varphi$. To see this, let $f$ be an arbitrary profile with $\rho(f) \models \varphi$. If $\rho(f) \not \models \gamma_{i}$, player $i$ would deviate by choosing the opposite value for $p$ in the first round. If, on the other hand, $\rho(f) \mid=\gamma_{i}$, player $j$ would deviate by choosing the opposite value for $q$ in the first round. Hence, $\left.N E(G)\right|_{\Phi} \subseteq \operatorname{runs}_{\Phi}(\neg \varphi)$. To see that this inclusion also holds in the opposite direction, assume that $\rho \not \models \varphi$ and that profile $f$ induces $\rho$. Since, neither $p$ nor $q$ occurs in $\varphi$, we may assume without loss of generality that $\rho \models \bar{p} \leftrightarrow q$. Therefore, player $j$ has its goal achieved and will not deviate. As, moreover, player $i$ controls no variables occurring in $\varphi$, no deviation from $f$ will satisfy her goal. It follows that $f$ is a Nash equilibrium and $\rho \in N E(G)$, as desired.

Previously, we have shown how LTL is equally expressive in formulas as it is in equilibrium. This required a rather elaborate proof, especially for LTL-expressible properties being non-countable. We find that the analogous result for projective expressiveness of LTL can now be show rather straightforwardly by reference to Propositions 5.1, 4.7, and 5.4, and Lemma 5.2.

THEOREM 5.6. Let $X \subseteq$ runs $_{\Phi}$ be a temporal property. Then, LTL can projectively express $X$ in formulas if and only if LTL can projectively express $X$ in equilibrium. Hence, LTL can projectively express in equilibrium all and only all $\omega$-regular temporal properties.

PROOF. In virtue of Propositions 5.1 and 4.7, it suffices to prove the first statement. The "only if"-direction follows immediately from Proposition 5.4. For the "if"-direction, assume that LTL can projectively express $X$ in equilibrium. Then, there is a set $\Psi$ of auxiliary propositional variables and an $\operatorname{LTL}(\Phi \cup \Psi)$-game $G$ such that $\left.N E(G)\right|_{\Phi}=X$. By Proposition 4.7, we know that the temporal property $N E(G)$ is $\omega$-regular. Lemma 5.2 then yields that $\left.N E(G)\right|_{\Phi}$ is $\omega$-regular property as well. In virtue of Proposition 5.1, we may conclude that LTL can projectively express $X=\left.N E(G)\right|_{\Phi}$ in formulas.

\subsection{The Next-free Fragment $\mathscr{L}_{U}$ Revisited}

In Section 4.4 , we saw that $\mathscr{L}_{U}$ can express in equilibrium some stutter-sensitive properties-in particular toggle $(p)$ - something it cannot do in formulas. On the other hand, we also found that the expressive power of $\mathscr{L}_{U}$ in equilibrium does not quite match the expressive power of full LTL in formulas (or, in reference to Theorem 4.8, that of LTL in equilibrium for that matter). In this section, however, we show that projectively $\mathscr{L}_{U}$ can express in equilibrium every temporal property 
that LTL can express projectively in equilibrium, that is, all and only all $\omega$-regular properties. This equivalence, however, does not hold for $\mathscr{L}_{U}$ 's projective expressiveness in formulas.

To appreciate the latter statement, it can easily be seen that the temporal properties that $\mathscr{L}_{U}$ can projectively express in formulas, are closed under 'positive' stuttering in the following sense. For every property $X$ that $\mathscr{L}_{U}$ projectively expresses in formulas, it holds that if run $v_{0} v_{1} v_{2} v_{3} \ldots$ is in $X$, so is $v_{0}^{k_{0}} v_{1}^{k_{1}} v_{2}^{k_{2}} v_{3}^{k_{3}} \ldots$ for all $k_{0}, k_{1}, k_{2}, \cdots \geq 1$. (Observe that this captures one half of the definition of stutter-invariance.) Let $X \subseteq$ runs $_{\Phi}$ be a property that $\mathscr{L}_{U}$ can projectively express in formulas. Then, there is some auxiliary set of variables $\Psi$ and some property $Y \subseteq \operatorname{runs}_{\Phi \cup \Psi}$ with $\left.Y\right|_{\Phi}=X$ that $\mathscr{L}_{U}$ can express in formulas. Now, let $v_{0} v_{1} v_{2} v_{3} \ldots$ be in $X$. Then, there is some run $w_{0} w_{1} w_{2} w_{3} \ldots$ in $Y$ such that $w_{t} \cap \Phi=v_{t}$ for all $t \geq 0$. As $\mathscr{L}_{U}(\Phi \cup \Psi)$ can express $Y$ in formulas, it follows that $w_{0}^{k^{0}} w_{1}^{k_{1}} w_{2}^{k_{2}} w_{3}^{k_{3}} \ldots$ must be in $Y$ as well. Then, moreover, it follows that $v_{0}^{k^{0}} v_{1}^{k_{1}} v_{2}^{k_{2}} v_{3}^{k_{3}} \ldots$ in $\left.Y\right|_{\Phi}=X$, as desired. Accordingly, $\mathscr{L}_{U}$ cannot projectively express in formulas some stutter-sensitive properties like toggle $(p)$.

The situation is quite different for the properties that $\mathscr{L}_{U}$ can projectively express in equilibrium, which, as the main result of this section, we find are exactly the $\omega$-regular ones. To obtain this result, we leverage the fact established by Proposition 4.9 that $\mathscr{L}_{U}$ can express in equilibrium the property toggle $(p)$.

Let $p$ be a variable not contained in $\Phi$. We first define the translation $\tau: \operatorname{LTL}(\Phi) \rightarrow \mathscr{L}_{\mathrm{U}}(\Phi \cup\{p\})$ inductively such that $q^{\tau}=q$ for all $q \in \Phi$, and

$$
\begin{aligned}
& (\neg \varphi)^{\tau}=\neg\left(\varphi^{\tau}\right) \\
& (\varphi \wedge \psi)^{\tau}=\varphi^{\tau} \wedge \psi^{\tau} \\
& (\varphi \vee \psi)^{\tau}=\varphi^{\tau} \vee \psi^{\tau} \\
& (\varphi \cup \psi)^{\tau}=\varphi^{\tau} \cup \psi^{\tau} \\
& (\mathrm{X} \varphi)^{\tau}=\left(p \rightarrow p \mathrm{U}\left(\bar{p} \wedge \varphi^{\tau}\right)\right) \wedge\left(\bar{p} \rightarrow \bar{p} \mathrm{U}\left(p \wedge \varphi^{\tau}\right)\right) .
\end{aligned}
$$

Then, on every run $\rho \in \Phi \cup\{p\}$ with $\left.\rho\right|_{\{p\}}=(p \bar{p})^{\omega}$, each formula $\varphi$ and its translation $\varphi^{\tau}$ will have the same truth-value.

LEMMA 5.7. Let $\varphi$ be an $\operatorname{LTL}(\Phi)$-formula, $p$ a propositional variable not in $\Phi$, and $\rho=$ $v_{0} v_{1} v_{2} \ldots$ a run in $\operatorname{runs}_{\Phi \cup\{p\}}$ such that $\rho, t \models p$ if and only if $t$ is even. Then, $\rho \models \varphi$ if and only if $\rho \models \varphi^{\tau}$.

PROOF. The proof proceeds by structural induction on $\varphi$. The basis is immediate and the induction hypothesis covers all inductive cases apart from $\varphi=\mathrm{X} \psi$. Consider an arbitrary $t \geq 0$ and assume $\rho, t \models \mathrm{X} \psi$. Then, $\rho, t+1 \mid=\psi$ and by the induction hypothesis also $\rho, t+1 \models \psi^{\tau}$. Now either $\rho, t \models p$ or $\rho, t \models \bar{p}$. First assume the former. Then immediately $\rho, t=\bar{p} \rightarrow \bar{p} \cup\left(p \wedge \psi^{\tau}\right)$. Moreover, $\rho, t+1 \models \bar{p}$ by definition of $\rho$ and, therefore, $\rho, t=p \mathrm{U}\left(\bar{p} \wedge \psi^{\tau}\right)$ and also $\rho, t \models p \rightarrow\left(p \bigcup\left(\bar{p} \wedge \psi^{\tau}\right)\right)$. We may conclude that $\rho, t \models(\mathrm{X} \psi)^{\tau}$, as desired. The argument if $\rho, t=\bar{p}$ is analogous.

For the opposite direction, assume $\rho, t \not \models \mathrm{X} \psi$. Then, $\rho, t+1 \not \neq \psi$ and by the induction hypothesis $\rho, t+1 \not \models \psi^{\tau}$. Now, either $\rho, t=p$ or $\rho, t \models \bar{p}$. If the former, both $\rho, t \not \models \bar{p} \wedge \psi^{\tau}$ and $\rho, t+1 \not \models \bar{p} \wedge \psi^{\tau}$. It follows that $\rho, t \not \forall p \cup\left(\bar{p} \wedge \psi^{\tau}\right), \rho, t \not \models p \rightarrow\left(p \mathrm{U}\left(\bar{p} \wedge \psi^{\tau}\right)\right)$, and eventually $\rho, t \not \neq(\mathrm{X} \psi)^{\tau}$. As the argument showing that $\rho, t \models \bar{p}$ is analogous, we may conclude the proof.

To obtain the main result of this section, we construct for each LTL-formula $\varphi$ a four-player $\mathscr{L}_{U^{-}}$ game with four additional variables. Intuitively, two of the players play the "matching pennies"-like game $G_{\varphi^{\tau}}^{m p}$, as it was employed in Proposition 4.1. This guarantees that $\varphi^{\tau}$ holds at all and only the equilibrium runs of the game. The other two players play the game $G_{\text {toggle }(p)}$, which facilitated the proof of Proposition 4.9. This then ensures that an additional variable $p$ alternately assumes the truth values true and false, and thus that $\varphi^{\tau}$ evaluates as intended, namely, as equivalent to $\varphi$.

THEOREM 5.8. The fragment $\mathscr{L}_{\mathrm{U}}$ can projectively express in equilibrium every temporal property that LTL can express in formulas. 
PROOF. Let $X$ be a property expressible by LTL in formulas. Then there is some $\operatorname{LTL}(\Phi)$ formula $\varphi$ with $X=\left\{\rho \in\right.$ runs $\left._{\Phi}: \rho \mid=\varphi\right\}$. Let furthermore $\Psi=\{p, q, r, s\}$ a set of auxiliary variables disjoint from $\Phi$, and construct $\mathscr{L}_{\mathrm{U}}$-game $G$ on $\Phi \cup \Psi$ with four players, 1, 2, 3, and 4, such that

$$
\Phi_{1}=\{p\}, \quad \Phi_{2}=\{q\}, \quad \Phi_{3}=\Phi \cup\{r\}, \quad \Phi_{4}=\{s\} .
$$

Let the players' goals, moreover, be given by:

$$
\begin{aligned}
& \gamma_{1}=p \wedge \neg \gamma_{2} \\
& \gamma_{2}=\mathrm{F}((p q \wedge p q \cup p \bar{q}) \vee(\bar{p} q \wedge \bar{p} q \cup \bar{p} \bar{q}) \vee(p \bar{q} \wedge p \bar{q} \cup p q) \vee(\bar{p} \bar{q} \wedge \bar{p} \bar{q} \cup \bar{p} q)), \\
& \gamma_{3}=\varphi^{\tau} \vee(r \leftrightarrow s), \\
& \gamma_{4}=\varphi^{\tau} \vee(r \leftrightarrow \bar{s}) .
\end{aligned}
$$

Thus, players 1 and 2 play $G_{\text {toggle }(p)}$. Proposition 4.9 ensures that in every equilibrium run $\rho$ in $\operatorname{runs}_{\Phi \cup \Psi}$, we have $\rho, t=p$ if and only if $t$ is even. Accordingly, by virtue of Lemma 5.7, on all equilibrium runs $\rho$ in $N E(G)$, we have $\rho \models \varphi^{\tau}$ if and only if $\rho \models \varphi$.

Players 3 and 4 -quite independently from 1 and $2-$ play $G_{\varphi^{\tau}}^{m p}$. In virtue of Proposition 4.1, we obtain, for every run $\rho \in \operatorname{runs}_{\Phi \cup \Psi}$, that $\rho=\varphi^{\tau}$ if and only if $\rho$ is an equilibrium run in $N E(G)$. Hence, $\rho \models \varphi$ if and only if $\rho \in N E(G)$, for all runs $\rho \in \operatorname{runs}_{\Phi \cup \Psi}$. Finally, as $\varphi$ only depends on $\Phi$, we also have that $\left.\rho\right|_{\Phi}=\varphi^{\tau}$ for every equilibrium run $\rho \in N E(G)$, and $\left.\rho\right|_{\Phi} \not \models \varphi^{\tau}$ for every non-equilibrium run $\rho \notin N E(G)$. It follows that $\left.N E(G)\right|_{\Phi}=X$, as desired.

It is worth noting that the size of $\varphi^{\tau}$ is exponential in the number of nestings of the X-operator, that is, even if $\mathscr{L}_{U}$ can projectively express every LTL-property in equilibrium, this may come at the cost of having exponentially longer goals for the players. Whether this exponential blowup is inevitable, we leave as an open question.

COROLlaRY 5.9. Let $X \subseteq$ runs $_{\Phi}$ be a temporal property. Then, $\mathscr{L}_{U}$ can projectively express $X$ in equilibrium if and only if $X$ is $\omega$-regular.

PROOF.

The "only if"-direction, let $X$ be a temporal property in runs $s_{\Phi}$ that $\mathscr{L}_{\mathrm{U}}(\Phi)$ can projectively express in equilibrium. Then, $\operatorname{LTL}(\Phi)$ can also projectively express $X$ in equilibrium, and, by Theorem 5.6, it follows that $X$ is $\omega$-regular.

For the "only if"-direction, consider an arbitrary $\omega$-regular property $X \subseteq$ runs $s_{\Phi}$. By Proposition 5.1, we know that $\operatorname{LTL}(\Phi)$ can projectively express $X$ in formulas. Hence, there is a set $\Psi$ of auxiliary variables and a property $Y \subseteq$ runs $_{\Phi \cup \Psi}$ that $\operatorname{LTL}(\Phi \cup \Psi)$ can express in formulas with $X=\left.Y\right|_{\Phi}$. Theorem 5.8 then gives us that $\mathscr{L}_{U}(\Phi \cup \Psi)$ can projectively express $Y$ in equilibrium. Accordingly, there is an auxiliary set $\Theta$ of variables and a temporal property $Z \subseteq$ runs $_{\Phi \cup \Psi \cup \Theta}$ that is expressed by $\mathscr{L}_{\cup}(\Phi \cup \Psi \cup \Theta)$ in equilibrium with $\left.Z\right|_{\Phi \cup \Psi}=Y$. We may conclude the proof by observing that $X=\left.Z\right|_{\Phi}$. Hence, $\mathscr{L}_{\mathrm{U}}(\Phi)$ can projectively express in equilibrium property $X$, as desired.

\section{LOGICAL INCENTIVE ENGINEERING: WEAK EXPRESSIVENESS}

In this section, we consider another weaker notion of expressiveness pertaining to non-empty temporal properties, which we will refer to as weak expressiveness. Thus, a fragment $\mathscr{L}(\Phi)$ is said to weakly express (in formulas) the non-empty property $X$ if it can express in formulas a stronger non-empty property, that is, if there is a satisfiable formula $\varphi$ of $\mathscr{L}(\Phi)$ with $\operatorname{runs}_{\Phi}(\varphi) \subseteq X$.

The concept of weak expressiveness in formulas is much weaker than the standard notion of expressiveness in formulas, in the sense that, if a fragment can express a property, it can also weakly express this property. To appreciate how much weaker weak expressiveness is, consider the seminal Ultimately Periodic Model Theorem by Sistla and Clarke [Sistla and Clarke 1985] that every every satisfiable formula is satisfied on an ultimately periodic run. Here, A run $\rho=v_{0} v_{1} v_{2} v_{3} \ldots$ is 
said to be ultimately periodic if there are integers $s, p \geq 0$, called the starting index and period, respectively, such that for every $t \geq s$, we have that $v_{t}=v_{t+p}$.

THEOREM 6.1 ([SISTLA AND ClARKE 1985]). Every satisfiable LTL-formula is satisfied by an ultimately periodic run.

Consequently, for an LTL-fragment $\mathscr{L}$ to weakly express every non-empty LTL-property in formulas, it already suffices to be able to express $\{\rho\}$ for every ultimately periodic run $\rho$. Now observe that any given ultimately periodic run $\rho=v_{0} v_{1} v_{2} v_{3} \ldots$ over $2^{\Phi}$ with starting index $s$ and period $p$ is characterised by the formula

$$
\chi_{\rho}=\bigwedge_{0 \leq t \leq s+p} \mathrm{X}^{t} \chi_{v_{t}} \wedge \mathrm{X}^{s} \mathrm{G}\left(\bigwedge_{v \in 2^{\Phi}}\left(\chi_{v} \rightarrow \mathrm{X}^{p} \chi_{v}\right)\right),
$$

where

$$
\mathrm{X}^{k} \varphi=\underbrace{\mathrm{X} \cdots X}_{k \text { times }} \varphi
$$

Accordingly, we find that every LTL-fragment that is at least as expressive as $\mathscr{L}_{\mathrm{X}, \mathrm{F}, \mathrm{G}}$, can weakly express in formulas every property that LTL can express in formulas.

Analogously to the other concepts of expressiveness in this paper, we also introduce projective weak expressiveness in formulas, as well as weak expressiveness in equilibrium, and projective weak expressiveness in equilibrium. Formally, a fragment $\mathscr{L}(\Phi)$ is said to be able to projectively weakly express in formulas a non-empty property $X \subseteq \operatorname{runs}_{\Phi}$ if there is some finite set $\Psi$ of variables and some formula $\varphi \in \mathscr{L}(\Phi \cup \Psi)$ such that for every run $\rho \in$ runs $_{\Phi \cup \Psi}$ we have that $\rho \in \operatorname{runs}_{\Phi}(\varphi)$ implies $\left.\rho\right|_{\Phi} \in X$. Moreover, $\mathscr{L}(\Phi)$ is said to weakly expresses $X$ in equilibrium if there is an $\mathscr{L}(\Phi)$-game $G$ with $\emptyset \subsetneq X \subseteq N E(G)$. Finally, $\mathscr{L}(\Phi)$ projectively expresses $X$ in equilibrium if there is some finite set $\Psi$ of auxiliary variables and some $\mathscr{L}(\Phi \cup \Psi)$-game $G$ such that $X=\left.N E(G)\right|_{\Phi}$. Finally, $\mathscr{L}$ projectively weakly expresses $X$ in equilibrium if there is some finite set $\Psi$ of auxiliary variables and some $\mathscr{L}$-game $G$ with the players's goals defined over the variables $\Phi \cup \Psi$ such that $\left.\varnothing \subsetneq X \subseteq N E(G)\right|_{\Phi}$.

These weak expressiveness notions mostly pertains are quite natural in settings where a designer uses temporal logic to specify the desired behaviour of a system. For instance, suppose a designer is to design a multi-agent system that is to behave accordingly to a given specification. If she manages to do so in such a way that the system behaves (in equilibrium) according to a stronger but consistent specification, she should still be satisfied: the additional features for the runs generated can be seen as belonging to the specifics of the implementation. Furthermore, in designing her system the designer may aim to distribute a possibly complex task over several agents each with limited computational capabilities by allocating to them tasks that are as "simple" as possible. The designer's objective as well as the specifications of the allocated tasks could be given by LTL-formulas in different fragments of LTL. Recall the Rabbit Hunt in Example 1.2 in the introduction, where we saw how LTL-specification could be implemented by the Nash equilibrium of a game with players having safety objectives that could be expressed in the weaker fragment $\mathscr{L}_{\mathrm{F}, \mathrm{G}}$.

For the remainder of this section, we will focus on the very weak fragment $\mathscr{L}_{\mathrm{X}, \mathrm{F}^{+}}$, where the Foperator cannot occur within the scope of a negation. We find that also this, rather weak, fragment can express more in equilibrium than it can in formulas. First we have the following lemma, which intuitively says that every $\mathscr{L}_{\mathrm{XF}^{+}}$-formula will be satisfied after a finite number of rounds on every satisfying run. Formally, a temporal property $X \subseteq \operatorname{runs}_{\Phi}$ is tail-invariant if $\rho \in X$ implies the existence of a prefix $\pi \in \operatorname{prefix}(\rho)$ such that $\pi ; \rho^{\prime} \in X$ for all $\rho^{\prime} \in$ runs $_{\Phi}$.

PROPOSITION 6.2. Every temporal property $X \subseteq$ runs $_{\Phi}$ that can be expressed in $\mathscr{L}_{\mathrm{X}, \mathrm{F}^{+}}$is tailinvariant.

Proof Sketch of PROOF. Let $\varphi \in \mathscr{L}_{\mathrm{X}, \mathrm{F}^{+}}$. As the F-operator does not occurs within the scope of a negation symbol $\neg$, exploiting the equivalence of $\neg X \varphi$ and $X \neg \varphi$, we can transform $\varphi$ to an equivalent formula in which all negation symbols occur in front of propositional variables. Hence, 
we may assume that $\varphi$ is in this normal form. Consider an arbitrary run $\rho=v_{0} v_{1} v_{2} v_{3} \ldots$ in runs $_{\Phi}$ such that $\rho=\varphi$. We have to show that there is a prefix $\pi \in \operatorname{prefix}(\rho)$ such that $\pi ; \rho^{\prime} \models \varphi$ for all $\rho^{\prime} \in \operatorname{runs}_{\Phi}$. Define inductively for every formula $\psi \in \mathscr{L}_{\mathrm{X}, \mathrm{F}^{+}}$and every $t \geq 0$, the integer $\kappa_{\rho, t}(\psi)$ as follows:

$$
\begin{aligned}
\kappa_{\rho, t}(p) & =\kappa_{\rho, t}(\bar{p})=0 \\
\kappa_{\rho, t}\left(\chi_{1} \wedge \chi_{2}\right) & =\max \left(\kappa_{\rho, t}\left(\chi_{1}\right), \kappa_{\rho, t} \kappa_{\rho, t}\left(\chi_{2}\right)\right) \\
\kappa_{\rho, t}\left(\chi_{1} \vee \chi_{2}\right) & =\min \left(\kappa_{\rho, t}\left(\chi_{1}\right), \kappa_{\rho, t} \kappa_{\rho, t}\left(\chi_{2}\right)\right) \\
\kappa_{\rho, t}(\mathrm{X} \chi) & =\kappa_{\rho, t}(\chi)+1 \\
\kappa_{\rho, t}(\mathrm{~F} \chi) & = \begin{cases}t^{\prime}-t+\kappa_{\rho, t^{\prime}}(\chi) & \text { if } \rho, t \models \mathrm{F} \chi, \\
0 & \text { otherwise, }\end{cases}
\end{aligned}
$$

where $t^{\prime}=\min \left\{t^{\prime \prime} \geq t: \rho, t^{\prime \prime} \models \chi\right\}$. By a structural induction on $\psi$ it can then be shown that $\rho, t=\psi$ implies the existence of a prefix $\pi \in \operatorname{prefix}(\rho)$ with $\operatorname{length}(\pi) \leq t+\kappa_{\rho, t}(\psi)$ such that $\pi_{\psi} ; \rho^{\prime}, t=\psi$ for all $\rho^{\prime} \in$ runs $_{\Phi}$. This holds in particular for $\varphi$ and $t=0$, which yields the result.

The property defined by the LTL-formula $\mathrm{G} p$ may serve as the quintessential property that is not tail-invariant, and is neither expressible nor weakly expressible in $\mathscr{L}_{\mathrm{X}, \mathrm{F}^{+}}$. Observing that tailinvariance of $X \subseteq$ runs $_{\Phi}$ implies tail-invariance of $\left.X\right|_{\Psi}$ for every $\Psi \subseteq \Phi$, we may even conclude that $\mathrm{G} p$ cannot even be projectively expressed by $\mathscr{L}_{\mathrm{X}, \mathrm{F}^{+}}$. Yet, as $\mathrm{G} p$ is LTL-equivalent to $\neg \mathrm{F} \bar{p}$, in virtue of Proposition 5.5, we find that $\mathscr{L}_{\mathrm{X}, \mathrm{F}^{+}}$can projectively express $\mathrm{G} p$ in equilibrium. That is, $\mathscr{L}_{\mathrm{X}, \mathrm{F}^{+}}$can projectively express in equilibrium strictly more temporal properties than it can express projectively in formulas.

Leveraging the same ideas, along with the fact that every ultimately periodic run can be characterised in $\mathscr{L}_{\mathrm{X}, \mathrm{F}, \mathrm{G}}$ with only one occurrence of the G-operator, we also obtain the following expressiveness result for $\mathscr{L}_{\mathrm{X}, \mathrm{F}^{+}}$with respect to LTL.

THEOREM 6.3. The fragment $\mathscr{L}_{\mathrm{X}, \mathrm{F}^{+}}$can projectively weakly express in equilibrium every property that LTL can express in formulas.

PRoOF. Let $\varphi \in$ LTL. If $\varphi$ is unsatisfiable, we are done immediately, because as $p \wedge \neg p$ is a formula in $\mathscr{L}_{\mathrm{X}, \mathrm{F}^{+}}$. On the other hand, if $\varphi$ is satisfiable then by Theorem 6.1 , there is an ultimately periodic run $\rho=v_{0} v_{1} v_{2} v_{3} \ldots$ with starting index $s$ and period $p$ such that is characterised by the LTL-formula $\chi_{\rho}$ given by

$$
\bigwedge_{0 \leq t \leq s+p} \mathrm{X}^{t} \chi_{v_{t}} \wedge \mathrm{X}^{s} \mathrm{G}\left(\bigwedge_{v \in 2^{\Phi}}\left(\chi_{v} \rightarrow \mathrm{X}^{p} \chi_{v}\right)\right)
$$

By suitably applying the laws of propositional logic, the duality of $F$ and $G$, as well as the equivalence of $\neg \mathrm{X} \varphi$ and $\mathrm{X} \neg \varphi$, we find that the negation $\neg \chi_{\rho}$ of $\chi_{\rho}$ is equivalent to

$$
\bigvee_{0 \leq t \leq s+p} \mathrm{X}^{i} \neg \chi_{v_{i}} \vee \mathrm{X}^{s} \mathrm{~F}\left(\bigvee_{v \in 2^{\Phi}}\left(\chi_{v} \wedge \mathrm{X}^{p} \neg \chi_{v}\right)\right) \text {, }
$$

which is included in the fragment $\mathscr{L}_{\mathrm{X}, \mathrm{F}^{+}}$. By Proposition 5.5, we know that $\mathscr{L}_{\mathrm{X}, \mathrm{F}^{+}}$can therefore projectively express $\chi_{\rho}$ in equilibrium. Hence, $\mathscr{L}_{\mathrm{X}, \mathrm{F}^{+}}$can weakly projectively express $\varphi$ in equilibrium, as desired.

As a corollary of Theorem 6.3, we find that $\mathscr{L}_{\mathrm{X}, \mathrm{F}^{+}}$can even weakly express in equilibrium every $\omega$-regular expression. The proof is analogous to that of the "if"-direction of Corollary 5.9.

COROLLARY 6.4. The fragment $\mathscr{L}_{\mathrm{X}, \mathrm{F}^{+}}$can weakly projectively express $X$ in equilibrium every $\omega$-regular property.

ProOF. Consider an arbitrary $\omega$-regular property $X \subseteq$ runs $_{\Phi}$. By Proposition 5.1, we know that LTL can projectively express $X$ in formulas. Hence, there is a set $\Psi$ of auxiliary variables and a property $Y \subseteq$ runs $_{\Phi \cup \Psi}$ that LTL can express in formulas with $X=\left.Y\right|_{\Phi}$. Theorem 5.8 then gives us that $\mathscr{L}_{\mathrm{X}, \mathrm{F}^{+}}$can weakly projectively express $Y$ in equilibrium. Accordingly, there is an auxiliary set $\Theta$ 
of variables and a temporal property $Z \subseteq \operatorname{runs}_{\Phi \cup \Psi \cup \Theta}$ that is expressed by $\mathscr{L}_{\mathrm{X}, \mathrm{F}^{+}}$in equilibrium and that is such that $\left.Z\right|_{\Phi \cup \Psi}=Y$. We may conclude the proof by observing that $X=\left.Z\right|_{\Phi}$. Hence, $\mathscr{L}_{\mathrm{X}, \mathrm{F}^{+}}$ can weakly projectively express in equilibrium property $X$, as desired.

\section{RELATED WORK}

The expressive power of LTL and many of its syntactic fragments has been a research topic for decades, with work showing connections with other temporal logic languages as well as results classifying the power of LTL and its fragments [Rabinovich 2002; Strejček 2004]. The most basic and well known classifications are with respect to sub-languages, that is, LTL fragments where only some operators are allowed. However, more refined studies have also been conducted, for instance, LTL fragments with respect to the allowed number of propositional variables or the number of nested temporal operators [Demri and Schnoebelen 2002].

Most of these studies have focused not only in the expressive power of the resulting sublogics but also in the implications of imposing such restrictions in the complexity of the model checking and satisfiability problems of such sublogics. These studies have also made it possible to understand connections between LTL fragments and standard automata models over infinite words-which in turn also easily show how to define different automata-theoretic decision procedures for each LTL sublanguage at hand [Vardi and Wolper 1994].

Despite the very many studies about the expressive power of LTL and related sublanguages, to the best of our knowledge, there are no results on the expressive power of LTL or its fragments with respect to the classes of runs that can be sustained by some Nash equilibrium. In this paper, we address precisely that issue and provide the first known results in the literature. The results are rather promising: they show that even though some LTL sublanguage, say $\mathscr{L}_{1}$, may be strictly more expressive than other LTL sublanguage, say $\mathscr{L}_{2}$, when interpreted over the full class of $\omega$-regular runs, such two sublogics $\mathscr{L}_{1}$ and $\mathscr{L}_{2}$ become equi-expressive when interpreted over a class of runs that can be sustained by some Nash equilibrium in a given class of games, as many of our results show.

As this kind of result can usually only be obtained by adding extra propositional variables to the "weaker" language, we also studied the expressive power, and game-theoretic implications, of allowing languages interpreted over different sets of propositional variables (projective expressiveness). Again, the results were promising in the sense that they show that generally weaker LTL sublogics can be made as expressive as generally stronger LTL sublogics by the addition of fresh propositional variables to the weaker language, a notion that goes back to the 1950s [Beth 1953; Craig 1957] and has proven useful in a number of settings [Hodges 1993; Chang and Keisler 1990; Gheerbrant and ten Cate 2009; Halpern et al. 2009].

\section{FINAL REMARKS AND FUTURE RESEARCH}

In this paper, we proposed the concept of expressiveness in equilibrium for linear temporal logics. Thus, we have explored the temporal properties that are characterised by the equilibrium runs of iterated Boolean games, where the players' dichotomous preferences are represented by formulas in various fragments of Linear Time Logic (LTL). We also introduced weak and projective variations of this concept. See Figure 2 for a schematic overview of our main findings in Sections 4 and 5.

The Nash equilibria of an iterated Boolean game are fully determined by the goals of the players and the way control of the propositional variables is distributed over the players. In particular, they are not dependent on an additional underlying game structure-like, for instance, concurrent game structures [Alur et al. 2002]. This enabled us to focus on the logical aspects of Nash equilibrium and accordingly we formulated our research issue in terms of expressiveness.

Apart from specific fragments, the concept of expressiveness in equilibrium gives rise to a number of more abstract and conceptual questions for future research.

First, in this paper we focussed mainly on the concepts of expressiveness in equilibrium and projective expressiveness in equilibrium. To highlight their formal features and how these notions relate to concepts of expressiveness in formulas, we concentrated on the full fragment LTL and the 


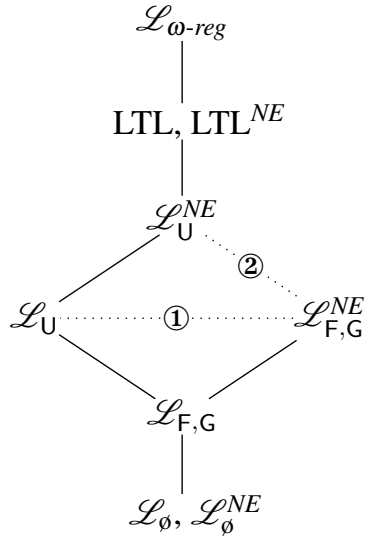

Expressiveness

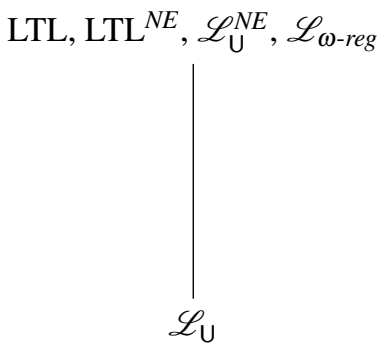

Projective expressiveness

Fig. 2. Overview of our main Results. A continuous line between two nodes indicates that the higher fragment is strictly more expressive than the lower fragment. Dotted line indicated by (1): as $\mathscr{L}_{\mathrm{F}, \mathrm{G}}$ can express in equilibrium some stuttersensitive properties, it is not less expressive in equilibrium than $\mathscr{L}_{\mathrm{U}}$ in formulas. We conjecture that there are also properties that $\mathscr{L}_{\mathrm{U}}$ can express in formulas that $\mathscr{L}_{\mathrm{F}, \mathrm{G}}$ cannot express in equilibrium, which would imply that $\mathscr{L}_{\mathrm{F}, \mathrm{G}}^{N E}$ and $\mathscr{L}_{\mathrm{U}}$ are incomparable. Dotted line indicated by (2): as $\mathscr{L}_{\mathrm{U}}$ is at least as expressive in formulas as $\mathscr{L}_{\mathrm{F}, \mathrm{G}}$ is in formulas, by virtue of Proposition 4.3 , we know that $\mathscr{L}_{\mathrm{U}}$ is also at least as expressive in equilibria as $\mathscr{L}_{\mathrm{F}, \mathrm{G}}$ is in equilibrium. We conjecture that $\mathscr{L}_{\mathrm{U}}$ is strictly more expressive in equilibria than $\mathscr{L}_{\mathrm{F}, \mathrm{G}}$ is in equilibrium.

important maximal stutter-invariant fragment $\mathscr{L}_{\mathrm{U}}$. To obtain a complete picture of (projective) expressiveness in equilibrium, we aim to explore a wider range of fragments. To this end, the hierarchy of temporal properties as proposed by [Manna and Pnueli 1990], [Chang et al. 1992], and [Černa and Pelánek 2003] may provide the necessary structure to this research. In particular, this hierarchy would allow us to address in a principled fashion fragments that can express safety, guarantee, obligation, response, persistence, and reactivity properties [Strejček 2004].

Second, another theoretical issue concerns the closure of the $\omega$-languages expressible in equilibrium under such operations as complement, intersection, and union. For some fragments, like full LTL and propositional calculus, the issue is trivial because these fragments are equally expressive in formulas as they are in equilibrium. For other fragments, like, for instance, the maximal stutter-invariant fragment $\mathscr{L}_{\mathrm{U}}$, the question is still very much open.

Third, in this paper we considered expressiveness in terms of $\omega$-runs. There is, however, also a considerable line of research that concerns the expressiveness of temporal logics when semantically interpreted on finite runs [Giacomo and Vardi 2013]. The model of iterated Boolean games can likewise be adapted in such a way that their outcomes are finite runs rather than infinite runs, as, for instance, in [Gutierrez et al. 2017b]. In that way, expressiveness in formulas and expressiveness in equilibrium with respect to finite runs can be compared. This would potentially allow us to leverage the work on forbidden fragments as originally introduced by [Cohen et al. 1993], which pertains to finite runs only and which furnishes us with some interesting characterisations of LTL-fragments [Strejček 2004].

Fourth, many of our game constructions involve a "matching pennies" game like $G_{\varphi}^{m p}$. These games establish a crucial link between runs that satisfy a given formula and equilibrium runs in iterated Boolean games. This feature, however, is due to one player trying to achieve $p \leftrightarrow q$ and another $p \leftrightarrow \bar{q}$, and as such is largely of a non-temporal nature. An interesting question is if this is peculiar to the results in this paper or that it points at a more fundamental connection with the concept of expressiveness in equilibrium. A related question for future research concerns (bounds 
on) the minimal number of players and additional propositional variables that may be needed to express or projectively express temporal properties in a particular fragment. In particular, it would be interesting to know if every property that can be expressed by a fragment $\mathscr{L}$ in equilibrium coincides with the equilibrium runs of a two-player $\mathscr{L}$-game.

Fifth, as in most work on model checking and concurrent game structures, the strategies of the players are functions from histories to choices whereas, in stark contrast, the preferences of the players are represented by logical formulas. As such the former are much finer grained than the latter. One may wonder to what extent the gap between expressiveness in formulas and expressiveness in equilibrium can be attributed to this "mismatch." For instance, would the phenomenon also occur in iterated Boolean games, if the players' strategies or abilities are similarly specified by temporal formulas?

Sixth, all our notions of expressiveness naturally extend to game-theoretic solution concepts other than Nash Equilibrium. Investigating some of these concepts is a natural line of future research.

Finally, in Section 6 we briefly dwelt on the topic of allocating tasks to agents so that a given property is satisfied in all resulting equilibria. We hope that our study of expressiveness in equilibrium has shed some light on this issue and the issue of to what extent the design of multi-agent systems in practice can be simplified by providing "lean" temporal specifications of individual agents who can be assumed to play strategies that together form an equilibrium.

Acknowledgements. This is an extensively revised and reorganised version of our paper that was presented at the 15th International Conference on Autonomous Agents and Multiagent Systems (AAMAS'16) [Gutierrez et al. 2016]. All authors acknowledge with gratitude the financial support of ERC Advanced Investigator Grant 291528 ("RACE") at the University of Oxford. Paul Harrenstein was also supported in part by ERC Starting Grant 639945 ("ACCORD") also at the University of Oxford. Michael Wooldridge and Paul Harrenstein furthermore acknowledge the financial support of the Alan Turing Institute in London. Giuseppe Perelli knowledges the support of the project "dSynMA", funded by the ERC under the European Union's Horizon 2020 Research and Innovation Programme (Grant Agreement No. 772459). The authors would also like to thank Johan van Benthem and Moshe Vardi for helpful discussions and insightful suggestions.

\section{REFERENCES}

R. Alur, T. A. Henzinger, and O. Kupferman. 2002. Alternating-time temporal logic. J. ACM 49, 5 (Sept. 2002), 672-713.

C. Baier and J.-P. Katoen. 2008. Principles of Model Checking. MIT Press.

E. W. Beth. 1953. On Padoa's Method in the Theory of Definition. Indagationes Mathematicae 15 (1953), 330-339.

E. Bonzon, M.-C. Lagasquie, J. Lang, and B. Zanuttini. 2006. Boolean games revisited. In Proceedings of the 17th European Conference on Artificial Intelligence (ECAI'06), G. Brewka, S. Coradeschi, A. Perini, and P. Traverso (Eds.). 265-269.

N. Bulling, W. Jamroga, and J. Dix. 2008. Reasoning about temporal properties of rational play. Annals of Mathematics and Artificial Intelligence 53, 1 (2008), 51-114.

I. Černa and R. Pelánek. 2003. Relating Hierarchy of Temporal Properties to Model Checking. In Proceedings of the 30th Symposium on the Mathematical Foundations of Computer Science (MFCS'03) (Lecture Notes in Computer Science (LNCS)), B. Rovan and Peter Vojtáš (Eds.), Vol. 2747. Springer-Verlag, 318-327.

C. C. Chang and H. J. Keisler. 1990. Model Theory (third ed.). Studies in Logic and the Foundations of Mathematics, Vol. 73. North-Holland Publishing Company.

E. Chang, Z. Manna, and A. Pnueli. 1992. Characterization of Temporal Property Classes. In Proceedings of the 19th International Colloquium on Automata, Languages and Programming (ICALP'92) (Lecture Notes in Computer Science (LNCS)), W. Kuich (Ed.), Vol. 623. Springer-Verlag, 474-486.

K. Chatterjee, T. A. Henzinger, and N. Piterman. 2010. Strategy Logic. Information and Computation 208, 6 (June 2010), 677-693.

E. M. Clarke, O. Grumberg, and D. A. Peled. 2000. Model Checking. The MIT Press: Cambridge, MA.

J. Cohen, D. Perrin, and J-É. Pin. 1993. On the Expressive Power of Termporal Logic. J. Comput. System Sci. 46 (1993), 271-294.

W. Craig. 1957. Three Uses of the Herbrand-Gentzen Theorem in Relating Model Theory and Proof Theory. Journal of Symbolic Logic 22, 3 (1957), 269-285. 
S. Demri, V. Goranko, and M. Lange. 2016. Temporal Logics in Computer Science: Finite State Systems. Cambridge Tracts in Theoretical Computer Science, Vol. 58. Cambridge University Press.

S. Demri and P. Schnoebelen. 2002. The Complexity of Propositional Linear Temporal Logics in Simple Cases. Information and Computation 174, 1 (2002), 84-103.

E. A. Emerson. 1990. Temporal and Modal Logic. In Handbook of Theoretical Computer Science Volume B: Formal Models and Semantics. Elsevier Science Publishers, 996-1072.

E. A. Emerson and J. Y. Halpern. 1986. 'Sometimes' and 'Not Never' Revisited: on Branching Time versus Linear Time Temporal Logic. J. ACM 33, 1 (1986), 151-178.

U. Endriss, S. Kraus, J. Lang, and M. Wooldridge. 2011. Designing Incentives for Boolean Games. In The 10th International Conference on Autonomous Agents and Multiagent Systems (AAMAS'11), K. Turner, P. Yolum, L. Sonenberg, and P. Stone (Eds.), Vol. 1. 79-86.

K. Etessami. 2000. A note on a question of Peled and Wilke on stutter-invariant LTL. Inform. Process. Lett. 75, 6 (2000), 261-263.

A. P. Gheerbrant and B. ten Cate. 2009. Craig Interpolation for Linear Temporal Languages. In Proceedings of 23rd international Workshop, CSL 2009, 18th Annual Conference of the EACSL, Coimbra, Portugal, September 7-11, 2009 (Lecture Notes in Computer Science), E Grädel and R. Kahle (Eds.), Vol. 5771. Springer, 287-301.

G. Giacomo and M. Vardi. 2013. Linear temporal logic and linear dynamic logic on finite traces. In Proceedings of the 23rd International Joint Conference on Artificial Intelligence (IJCAI'13), S Abramsky, G Gottlob, and PG Kolaitis (Eds.). 854-860.

J. Gutierrez, P. Harrenstein, G. Perelli, and M. Wooldridge. 2016. Expressiveness and Nash Equilibrium in Iterated Boolean Games. In Proceedings of the 15th International Conference on Autonomous Agents \& Multiagent Systems (AAMAS'16), J. Thangarajah, K. Tuyls, C. Jonker, and S. Marsella (Eds.). 707-715.

J. Gutierrez, P. Harrenstein, and M. Wooldridge. 2013. Iterated Boolean Games. In Proceedings of the 23rd International Joint Conference on Artificial Intelligence (IJCAI'13), F. Rossi (Ed.). 932-938.

J. Gutierrez, P. Harrenstein, and M. Wooldridge. 2014. Reasoning about equilibria in game-like concurrent systems. In 14th Conference on Principles of Knowledge Representation and Reasoning (KR'14). 408-417.

J. Gutierrez, P. Harrenstein, and M. Wooldridge. 2015a. Expressiveness and Complexity Results for Strategic Reasoning. In CONCUR'15 (LIPICs), Vol. 42. Schloss Dagstuhl, 268-282.

Julian Gutierrez, Paul Harrenstein, and Michael Wooldridge. 2015b. Iterated Boolean games. Information and Computation 242 (2015), 53-79.

J. Gutierrez, P. Harrenstein, and M. Wooldridge. 2017a. Reasoning about equilibria in game-like concurrent systems. Annals of Pure and Applied Logic 168, 2 (2017), 373-403.

J. Gutierrez, G. Perelli, and M. Wooldridge. 2017b. Iterated Games with LDL Goals over Finite Traces. In Proceedings of the 16th Conference on Autonomous Agents and MultiAgent Systems (AAMAS'17). 696-704.

J. Y. Halpern, D. Samet, and E. Segev. 2009. On Definability in Multimodal Logic. The Review of Symbolic Logic 2, 3 (2009), 451-468.

P. Harrenstein, W. van der Hoek, J.-J.Ch. Meyer, and C. Witteveen. 2001. Boolean Games. In Proceeding of the Eighth Conference on Theoretical Aspects of Rationality and Knowledge (TARK VIII), J. van Benthem (Ed.). Siena, Italy, 287-298.

W. Hodges. 1993. Model Theory. Encyclopedia of Mathematics and its Applications, Vol. 42. Cambridge University Press.

O. Kupferman. 2018. Automata Theory and Model Checking. In Handbook of Model Checking, Edmund M. Clarke, Thomas A. Henzinger, Helmut Veith, and Roderick Bloem (Eds.). Springer-Verlag, 107-151.

A. Kučera and J. Strejček. 2005. The stuttering principle revisited. Acta Informatica 41, 7-8 (2005), $415-434$.

L. Lamport. 1983. What Good is Temporal Logic?. In Information Processing '83: Proceedings of the 9th IFIP Congress on Information Processing, R. E. A. Mason (Ed.). North-Holland, 657-667.

Christof Löding. 2012. Basics on Tree Automata. In Modern Applications of Automata Theory, D. D'Souza and P Shankar (Eds.). IISc Research Monographs Series, Vol. 2. World Scientific, 79-110.

R. D. Luce and H. Raiffa. 1957. Games and Decisions. John Wiley \& Sons.

Z. Manna and A. Pnueli. 1990. A Hierarchy of Temporal Properties. In Proceedings of the ACM Symposium on Principels of Distributed Computing (PODC'90). ACM Press, 377-410.

M. Maschler, E. Solan, and S. Zamir. 2013. Game Theory. Cambridge U.P.

A. Mateescu and A. Salomaa. 1997. Formal Languages: An Introduction and a Synopsis. In Handbook of Formal Languages, G. Rozenberg and A. Salomaa (Eds.). Vol. I: Word, Language, Grammar. Springer, Chapter 1.

D. Niwinski and I. Walukiewicz. 1998. Relating Hierarchies of Word and Tree Automata. In Annual Symposium on Theoretical Aspects of Computer Science 1998 (STACS'98) (Lecture Notes in Computer Science (LNCS)), Vol. 1373. Springer, 320-331.

M. J. Osborne and A. Rubinstein. 1994. A Course in Game Theory. The MIT Press: Cambridge, MA. 
D. Peled. 1997. On Projective and Separable Properties. Theoretical Computer Science 186 (1997), 135-157.

D. A. Peled and T. Wilke. 1997. Stutter-invariant temporal properties are expressible without next-time operator. Inform. Process. Lett. 63, 5 (1997), 243-246.

D. Perrin and J-É. Pin. 2004. Infinite Words: Automata, Semigroups, Logic and Games. Pure and Applied Mathematics, Vol. 141. Elsevier Academic Press.

A. Pnueli. 1977. The Temporal Logic of Programs. In Proceedings of the Eighteenth IEEE Symposium on the Foundations of Computer Science. 46-57.

A. Pnueli and R. Rosner. 1989. On the Synthesis of a Reactive Module. In Proceedings of the Sixteenth ACM Symposium on the Principles of Programming Languages (POPL). 179-190.

Alexander Moshe Rabinovich. 2002. Expressive Power of Temporal Logics. In CONCUR 2002 - Concurrency Theory, 13th International Conference, Brno, Czech Republic, August 20-23, 2002, Proceedings (Lecture Notes in Computer Science), Lubos Brim, Petr Jancar, Mojmír Kretínský, and Antonín Kucera (Eds.), Vol. 2421. Springer, 57-75.

Y. Shoham and K. Leyton-Brown. 2008. Multiagent Systems: Algorithmic, Game-Theoretic, and Logical Foundations. Cambridge University Press: Cambridge, England.

A. P. Sistla and E. M. Clarke. 1985. The Complexity of Propositional Linear Temporal Logics. J. ACM 32, 3 (1985), 733-749.

J. Strejček. 2004. Linear Temporal Logic: Expressiveness and Model Checking. Ph.D. Dissertation. Masaryk University of Brno.

W. Thomas. 1990. Automata on Infinite Objects. In Handbook of Theoretical Computer Science Volume B: Formal Models and Semantics. Elsevier Science Publishers B.V.: Amsterdam, The Netherlands, 133-192.

W. Thomas. 1997. Languages, Automata, and Logic. In Handbook of Formal Languages, G. Rozenberg and A. Salomaa (Eds.). Vol. III: Beyond words. Springer, Chapter 5.

M. Y. Vardi. 1996. An Automata-theoretic Approach to Linear Temporal Logic. In Logics for Concurrency: Structure vs Automata, A. Moller and G. Birtwistle (Eds.). Lecture Notes in Computer Science (LNCS), Vol. 1043. Springer, 238266.

M. Y. Vardi. 2001. Branching vs. Linear Time: Final Showdown. In Proceedings of the 2001 Conference on Tools and Algorithms for the Construction and Analysis of Systems (TACAS'01), T. Margaria and W. Yi (Eds.), Vol. 2031. Lecture Notes in Computer Science, 1-22.

M. Y. Vardi and P. Wolper. 1994. Reasoning about Infinite Computations. Information and Computation 115, 1 (1994), 1-37.

P. Wolper. 1983. Temporal Logic Can Be More Expressive. Information and Control 56, 1-2 (1983), 72-99. 